\title{
NUMERICAL METHODS FOR COMPUTING NONLINEAR EIGENPAIRS: PART II. NON ISO-HOMOGENEOUS CASES
}

\author{
XUDONG YAO* AND JIANXIN ZHOU ${ }^{\dagger}$
}

\begin{abstract}
Standing (solitary)-wave/steady-state solutions in many nonlinear wave motions and Schrodinger flows lead to nonlinear eigenproblems. In [X. Yao and J. Zhou, SIAM J. Sci. Comput., 29 (2007), pp. 1355-1374], a Rayleigh-local minimax method is developed to solve iso-homogeneous eigenproblems. In this subsequent paper, a unified method in Banach spaces is developed for solving non iso-homogeneous even non homogeneous eigenproblems and applied to solve two models: the Gross-Pitaevskii problem in the Bose-Einstein condensate and the p-Laplacian problem in nonNewtonian flows/materials. First a new active Lagrange functional is formulated to establish a local minimax characterization. A local minimax method is then devised and implemented to solve the model problems. Numerical results are presented. Convergence results of the algorithm and an order of eigensolutions computed by the algorithm are also established.
\end{abstract}

Key words. Nonlinear eigenproblems, Critical points, Minimax method, active Lagrange multiplier

AMS subject classifications. 58E05, 58E30,35A40,35J65

1. Introduction. To study pattern formation, (in)stability and other solution properties in a wave motion

$$
w_{t t}=\Delta w+\kappa f(|w|) w
$$

or a "Schrodinger flow"

$$
-i w_{t}=\Delta w+\kappa f(|w|) w
$$

where $\kappa$ is a physical parameter, the standing (solitary) wave solutions $w(x, t)=$ $u(x) e^{-i \lambda t}$, where $\lambda$ is a normalized potential and $u$ is a function of $x$, and also the steady-state solutions $w(x, t)=u(x)$ are particularly interested $[5,17,26]$. Then $(1.1)$ and (1.2) lead to two semilinear elliptic PDEs

$$
\begin{aligned}
& \Delta u(x)+\kappa f(|u|) u(x)=-\mu u(x), \\
& \Delta u(x)+\kappa f(|u|) u(x)=0
\end{aligned}
$$

where $\mu=\lambda^{2}$ or $\mu=\lambda$. When non Darcian/Newtonian fluids are considered, the Darcy's law is replaced by other relations between the specific discharge (shear stress) $\vec{q}$ and the flow head $w$. One of them is the Izbash formula $[13,6]: \vec{q}=-M|\nabla w|^{p-2} \nabla w$ for some constant $M>0$ and the (rheological) characteristic $p>1$ of the fluid. Then after replacing the Laplacian $\Delta u$ by the p-Laplacian $\Delta_{p} u(x)=\nabla \cdot\left(|\nabla u(x)|^{p-2} \nabla u(x)\right)$, where $\nabla u$ is the gradient of $u,|\nabla u|$ is its Euclidean norm in $\mathbb{R}^{n},(1.3)$ and (1.4) become two quasilinear elliptic PDEs

$$
\begin{aligned}
& -\Delta_{p} u(x)-\kappa f(|u(x)|) u(x)=\mu u(x), \\
& -\Delta_{p} u(x)=\kappa f(|u(x)|) u(x) .
\end{aligned}
$$

\footnotetext{
*Department of Mathematics, University of Connecticut, Storrs, CT 06269. Presently at Department of Mathematics, University of Arkansas, Fayetteville, AR 72701, xyao@uark.edu

$\dagger$ Department of Mathematics, Texas A\&M University, College Station, TX 77843, jzhou@math.tamu.edu. This author's research is supported in part by NSF DMS-0311905
} 
When $p=2, p<2, p>2$, the fluid is called, respectively, (Darcian) Newtonian, pseudo-plastic, dilatant. People are interested in knowing for what values of $\mu$ and $\kappa,(1.5)$ and (1.6) have nontrivial, positive or nodal solutions. To develop a unified approach for solving the problems, we consider the following variational nonlinear eigenproblem (NEP): for given $\alpha>0$, find eigenpairs, called eigensolutions, $(\lambda, u) \in$ $\mathbb{R} \times B$ s.t.

$$
F^{\prime}(u)=\lambda G^{\prime}(u) \quad \text { in } \quad B^{\prime} \text { subject to } \quad G(u)=\alpha
$$

where $B$ is a Banach space with the norm $\|\cdot\|, B^{\prime}$ its dual, $F^{\prime}$ and $G^{\prime}$ are the Frechet derivatives of functionals $F$ and $G$ in $C^{1}(B, \mathbb{R}), \lambda$ is called an eigenvalue and $u$, which can be either real or complex-valued, is called an eigenfunction corresponding to $\lambda$. We refer to $[1,3,4,7,17]$ for more applications and theoretical studies of (1.7). The simplest case is the eigenproblem in a Hilbert space $B$ :

$$
F(u)=\frac{1}{2}\langle A u, u\rangle, \quad G(u)=\frac{1}{2}\langle u, u\rangle,
$$

where $A$ is a linear self-adjoint operator from $B$ to $B$ and $\langle$,$\rangle is the inner product.$ Thus both $F^{\prime}$ and $G^{\prime}$ are linear. For this problem, its wide applications are well known and many numerical packages are available. When either $F^{\prime}$ or $G^{\prime}$ is nonlinear, (1.7) is a NEP. Recall that an NEP is homogeneous if there are integers $m, l$ s.t.

$$
F^{\prime}(t u)=t^{m-1} F^{\prime}(u) \text { and } G^{\prime}(t u)=t^{l-1} G^{\prime}(u), \forall t>0, u \in B .
$$

If (1.9) is satisfied with $m=l$ (a resonance case), then the NEP is called isohomogeneous. The well known NEP of the p-Laplacian $\Delta_{p}$ and the Monge-Ampére operator $M$ in $[26,20]$ are iso-homogeneous. A Rayleigh-local minimax method (RLMM) is developed in [26] to solve this class of NEP for multiple eigensolutions in the order of their eigenvalues.

As a subsequent paper, here we develop a unified method to solve more general, non iso-homogeneous even non homogeneous eigenproblems of (1.5) and (1.6) for multiple solutions in certain sequential order. In particular, we consider two model problems:

Model Problem 1: The Gross-Pitaevskii equation [2, 8, 9, 11,18]

(1.10) $i w_{t}(x, t)=-\frac{1}{2} \Delta w(x, t)+V(x) w(x, t)+\beta|w(x, t)|^{2} w(x, t), \quad t>0, x \in \Omega \subseteq \mathbb{R}^{d}$,

(1.11) $w(x, t)=0, \quad x \in \Gamma=\partial \Omega, t \geq 0$,

in a bounded open domain $\Omega \in \mathbb{R}^{d}$, is used extensively as a mathematical model to describe the single particle properties of the Bose-Einstein condensate, where $w$ is the macroscopic wave function of the condensate, $t$ is the time, $x$ is the spatial coordinate, $V(x)$ is a trapping potential which is usually harmonic and can be written as $V(x)=$ $\frac{1}{2}\left(\gamma_{1}^{2} x_{1}^{2}+\cdots+\gamma_{d}^{2} x_{d}^{2}\right)$ with $\gamma_{1}, \ldots, \gamma_{d}>0$, and $\beta>0$ measures a repulsive nonlinearity. This model has been shown a great agreement with experimental realization of the Bose-Einstein condensate in several cases, e.g., the ground states of the condensate [19], free expansion [12] and lower energy excitations [9]. Note that the original model is set for solutions $w$ on the entire space $\mathbb{R}^{d}$ under a localized property that $w(x, t) \rightarrow 0$ as $\|x\| \rightarrow \infty$. Thus the solutions $w$ can be considered to satisfy the zero Dirichlet boundary condition on a sufficiently large spherical or cubic domain. Then due to the special form of $V$, with a proper scaling on $w$ and $V(x)$, the problem can be set on 
a unit spherical or cubic domain. An important invariant is the normalization of the wave-function

$$
\int_{\Omega}|w(x, t)|^{2} d x=1
$$

Under (1.12), finding the solitary wave solutions $w(x, t)=u(x) e^{-i \mu t}$ to (1.10) leads to

$$
\mu u(x)=-\frac{1}{2} \Delta u(x)+V(x) u(x)+\beta|u(x)|^{2} u(x), \quad x \in \Omega \text { s.t. } \int_{\Omega}|u(x)|^{2} d x=1,
$$

a non homogeneous NEP for eigensolutions $(\mu, u) \in \mathbb{R} \times W_{0}^{1,2}(\Omega)$. Many researchers have tried to numerically solve (1.13), see $[10,8,2]$ and references therewith. Also in [23], in addition to proposing new algorithms to solve (1.13) and proving their local convergence, the authors presented a comparison of existing algorithms to solve (1.13).

Model Problem 2: Consider NEP of finding $(\lambda, u) \in \mathbb{R} \times W^{1, p}(\Omega) \backslash\{0\}$ s.t.

$$
-\Delta_{p} u(x)=\lambda w(x)|u(x)|^{q-2} u(x), x \in \Omega,\left.u(x)\right|_{\partial \Omega}=0 \text {, s.t. } \int_{\Omega} w(x)|u(x)|^{q} d x=1,
$$

in an open bounded domain $\Omega \subset \mathbb{R}^{n}$, where $w$ is a (generalized) weight function. Such a NEP appears in study of non-Newtonian fluids/materials $[5,6,17]$. It is clear that $\operatorname{NEP}(1.14)(p \neq 2, q \neq 2)$ is homogeneous but non iso-homogeneous when $p \neq q$.

Due to the general space setting, nonlinearity of the problem and multiplicity of solutions, (1.7) is extremely difficult to solve. So far a reasonably general and mathematically justified numerical algorithm to solve (1.7) for multiple eigensolutions in an order is still not available, although several existence results of multiple solutions to (1.7) are established, see Proposition 44.26 in [27] and results in [14]. It is natural to define its Lagrange functional

$$
\mathcal{L}(\lambda, u)=F(u)-\lambda(G(u)-\alpha) .
$$

Then $(\bar{\lambda}, \bar{u})$ is an eigensolutions of (1.7) iff $(\bar{\lambda}, \bar{u})$ is a critical point of (1.15), i.e., the Frechet derivative $\nabla \mathcal{L}(\bar{\lambda}, \bar{u})=0 . \quad c=\mathcal{L}(\bar{\lambda}, \bar{u})=F(\bar{u})$ is called the critical value of $(\bar{\lambda}, \bar{u})$. The first candidates for critical points are the local extrema of $\mathcal{L}$. Critical points that are not local extrema are called saddle points, which appear as unstable equilibria or excited states in a physical system. Thus their instability is an interesting information to application.

Computationally whether or not an algorithm can find eigenfunctions in certain order is an important issue. With an order, a successful numerical algorithm should be able to find at least the first few eigenfunctions. When (1.7) is linear in a Hilbert space as in (1.8), due to the orthogonality between different eigenfunctions, the Rayleigh-Ritz method [27] can be used to find eigenfunctions following the order of their eigenvalues. Can one do so when (1.7) is nonlinear and the orthogonality between different eigenfunctions no longer holds? Theoretically since all eigenvalues of (1.7) are assumed to be real, the eigenfunctions can always be ordered in the order of their eigenvalues. But the question is whether or not a numerical method can find eigenfunctions of (1.7) following the order of their eigenvalues. So far such a numerical algorithm is still not available. One may mention certain type of Newton's method or the newly proposed squared-operator iteration methods [23] for their fast 
local convergence. Theoretically they can find all the eigenfunctions as long as initial guesses are selected sufficiently close to desired unknown solutions. But the question is how can one select initial guesses sufficiently close to desired unknown solutions while solution patterns are still unknown for many nonlinear systems? In particular, how can one do so in an order? Note that those two types of methods do not assume or use the variational structure of a problem. Their final solutions depend strongly on initial guesses. Thus in general they alone are blind to an order of solutions. When they are applied to solve Model Problem 1 (semilinear), it becomes a problem of selecting initial guesses that are sufficiently close to desired unknown solutions in an order. The algorithm developed in this paper can also serve for such purpose [22]. However, we do not know how to apply those two types of methods to solve Model Problem 2 (quasilinear).

The local minimax method (LMM) developed in [15, 16, 28, 24, 25] can find critical points of a functional following the order of saddles and their critical values, which provides important information on the structural instability of a critical point. It is interesting if we know the relation between the order of critical values of (1.15) and the order of eigenvalues of (1.7). R-LMM developed in [26] can only solve an iso-homogeneous NEP in the order of their eigenvalues, which also coincide with their critical values. But many NEP in applications such as Model Problem $2(p \neq q)$ and Model Problem 1 appear as non iso-homogeneous even non homogeneous. Thus a new method must be developed to solve such problems.

It is known that in order for (1.15) to possess multiple critical points and for LMM to find them, the functional $\mathcal{L}$ in (1.15) needs to have certain structure, either a global one or a local one surrounded by a barrier. For example, $\mathcal{L}$ needs to have either a global mountain pass structure, i.e., for each $(\lambda, u) \in(\mathbb{R} \backslash\{0\}) \times(B \backslash\{0\})$

$$
t_{(\lambda, u)}=\arg \text { local- } \max _{t>0} \mathcal{L}(t(\lambda, u))>0
$$

or a local mountain pass structure, i.e., (1.16) holds for each $(\lambda, u)$ in an open set $U$ and $t_{(\lambda, u)} \rightarrow+\infty$ when $(\lambda, u) \in U$ and $(\lambda, u) \rightarrow \partial U$, the boundary of $U$. Since for most eigenproblems, (1.16) does not hold for $\lambda=0$, a global mountain pass structure cannot be expected for (1.15). Thus we assume only a local (mountain pass or linking) structure (See Remark 2.1 (c)). But even this is not always true for (1.15). So we replace (1.15) by a new and more general formulation, called an active Lagrange functional

$$
\mathcal{L}(\lambda, u)=F(u)-\lambda(k)(G(u)-\alpha),
$$

where $k \geq 1$ is a parameter and $\lambda(k)=|\lambda|^{k}$ (or other form) is called an active Lagrange multiplier. The merit of introducing an extra parameter $k$ in (1.17) is twofold: firstly, one may raise the value of $k$ to improve the functional structure. For example, when the growth order of $G(u)$ is one less than that of $F(u)$, for each $(\lambda, u) \in(\mathbb{R} \backslash\{0\}) \times(B \backslash\{0\}),(1.16)$ does not hold for (1.15), or (1.17) with $k=1$. Then we can raise the value of $k$ in (1.17) s.t. (1.16) holds for each of such $(\lambda, u)$, and LMM can be applied; secondly, one may also raise the value of $k$ to satisfy some assumption in a convergence result of our algorithm, see Theorem 4.8 and Lemma 4.9 where $k=\ell$. Then the following lemma is obvious.

Lemma 1.1. Assume $\bar{\lambda} \neq 0$. Then $(\bar{\lambda}, \bar{u})$ is a critical point of (1.17) iff $\left(|\bar{\lambda}|^{k}, \bar{u}\right)$ is an eigensolution of (1.7).I

For most NEP, $(0, u)$ is not a critical point of (1.17). Even it is, (1.17) does not 
provide any information about the constraint $G(u)=\alpha$ when $k>1$. Thus such case is not interested.

For (1.17), by assuming a local (linking) structure defined in Remark 2.1 (c), we develop, in Section 2, a new local minimax characterization of saddle points and its corresponding algorithm. In Section 3, we implement the algorithm to numerically solve the two model problems for multiple eigensolutions. Finally in Section 4, we present some theoretical results on the convergence of the algorithm and the order of eigensolutions found by the algorithm.

2. Local Minimax Characterization and Method. Let $\langle,\rangle_{p r}$ be the dual relation between $(\mathbb{R} \times B)^{*},\|(\lambda, u)\|_{p r}=\sqrt{|\lambda|^{2}+\|u\|^{2}}$ be the norm of each $(\lambda, u)$ in the product space $\mathbb{R} \times B$ and $\mathbb{R} \times B$ and $L_{p r}=\left[\left(\lambda_{1}, u_{1}\right), \ldots,\left(\lambda_{n-1}, u_{n-1}\right)\right]$ be the space spanned by $n-1$ linearly independent previously found critical points $\left(\lambda_{1}, u_{1}\right), \ldots,\left(\lambda_{n-1}, u_{n-1}\right) \in(\mathbb{R} \backslash\{0\}) \times(B \backslash\{0\})$. For each $(\lambda, u) \in \mathbb{R} \times B$, denote $\left[L_{p r},(\lambda, u)\right]=\left\{w=\sum_{k=1}^{n-1} t_{k}\left(\lambda_{k}, u_{k}\right)+t_{n}(\lambda, u) \mid t_{k} \in \mathbb{R}, k=1, \ldots, n\right\}$. Assume that $\mathbb{R} \times B=L_{p r} \oplus L_{p r}^{\prime}$ and the corresponding linear bounded projection $\mathcal{P}: \mathbb{R} \times B \rightarrow L_{p r}^{\prime}$, and the functional $J(\lambda, u)=\mathcal{L}(\lambda, u)$ in (1.17). For each subspace $A$ of $\mathbb{R} \times B$, denote $S_{A}$ the unit sphere of $A$.

2.1. A Local Minimax Characterization of Eigensolutions. Definition 2.1. Given an open set $U$ of $S_{L_{p r}^{\prime}}$. A set-valued mapping $P: U \rightarrow 2^{\mathbb{R} \times B}$ is the peak mapping of $J$ w.r.t. $L_{p r}$ if for each $(\lambda, u) \in U, P(\lambda, u)$ is the set of all local maximum points of $J$ in $\left[L_{p r},(\lambda, u)\right]$. A single-valued mapping $p: U \rightarrow \mathbb{R} \times B$ is a peak selection of $J$ w.r.t. $L_{p r}$ if $p(\lambda, u) \in P(\lambda, u), \forall(\lambda, u) \in U$.

REMARK 2.1. (a) The above definition of a peak selection can be generalized as below: Given an open set $U$ of $S_{L_{p r}^{\prime}}$. A set-valued mapping $P: U \rightarrow 2^{\mathbb{R} \times B}$ is the $L_{p r}-\perp$ mapping of $J$ if for each $(\lambda, u) \in U, P(\lambda, u)$ is the set of all points $(\mu, w) \in\left[L_{p r},(\lambda, u)\right]$ s.t. $\langle\nabla J(\mu, w),(\lambda, u)\rangle_{p r}=0$ and $\left\langle\nabla J(\mu, w),\left(\lambda_{i}, u_{i}\right)\right\rangle_{p r}=0$, $i=1,2, \ldots, n-1$. A single-valued mapping $p: U \rightarrow \mathbb{R} \times B$ is an $L_{p r}-\perp$ selection of $J$ if $p(\lambda, u) \in P(\lambda, u), \forall(\lambda, u) \in U$.

(b) It is clear that a peak selection is an $L_{p r}-\perp$ selection, and Lemma 2.3 and Theorem 2.4 can be verified in the same way if a peak selection $p$ is replaced by an $L_{p r}-\perp$ selection $p$.

(c) From now on we assume that $U \subset S_{L_{p r}^{\prime}}$ is open s.t. a peak ( $\left.L_{p r}-\perp\right)$ selection $p$ is defined in $U$ and $\|p(\lambda, u)\| \rightarrow+\infty$ for $(\lambda, u) \in U$ and $(\lambda, u) \rightarrow \partial U$. Such a local barrier structure is used for (1.17) to have and for LMM to find a critical point under consideration.

In a descent direction method, the gradient of a functional plays an important role in a Hilbert space. This role is replaced by a pseudo-gradient of a functional in a Banach space.

Definition 2.2. Let $X$ be a Banach space, $J \in C^{1}(X, \mathbb{R})$ and $u \in X$ be a point s.t. $\nabla J(u) \neq 0$. For given $\theta \in(0,1]$, a point $\Psi(u) \in X$ is a pseudo-gradient of $J$ at $u$ w.r.t. $\theta$ if

$$
\|\Psi(u)\| \leq 1, \quad\langle\nabla J(u), \Psi(u)\rangle \geq \theta\|\nabla J(u)\| .
$$

Denote $\hat{X}=\{u \in X: \nabla J(u) \neq 0\}$. A pseudo-gradient flow of $J$ with a constant $\theta$ is a continuous mapping $\Psi: \hat{X} \rightarrow X$ s.t. $\forall u \in \hat{X}, \Psi(u)$ satisfies (2.1). The following lemma leads to a local minimax characterization of saddle points and also provides a stepsize rule in Step 4 of the new algorithm. 
Lemma 2.3. Given $\gamma>0$. Assume that a peak selection $p$ of $J$ defined in $U$ is continuous at $\bar{v}=(\bar{\lambda}, \bar{u}) \in U$ with $\nabla J(p(\bar{v})) \neq 0$ and $p(\bar{v}) \notin L_{p r}$. When $s>0$ is small, we have $\bar{v}(s)=\frac{\bar{v}-s w(p(\bar{v}))}{\|\bar{v}-s w(p(\bar{v}))\|_{p r}} \in U$ s.t.

$$
s\|w(p(\bar{v}))\|_{p r}<\gamma \quad \text { and } \quad J(p(\bar{v}(s)))<J(p(\bar{v}))-\frac{s \theta}{4}\left|t_{n}\right|\|\nabla J(p(\bar{v}))\|_{p r}
$$

where $p(\bar{v})=\sum_{i=1}^{n-1} t_{i}\left(\lambda_{i}, u_{i}\right)+t_{n} \bar{v}, w(p(\bar{v}))=\operatorname{sign}\left(t_{n}\right) \mathcal{P}(G(p(\bar{v})))$ and $G(p(\bar{v}))$ is a pseudo-gradient of $J$ at $p(\bar{v})$ with constant $\theta \in(0,1)$.

Proof. Note that

$$
p(\bar{v}(s)) \equiv p\left(\frac{\bar{v}-s w(p(\bar{v}))}{\|\bar{v}-s w(p(\bar{v}))\|_{p r}}\right)=\sum_{i=1}^{n-1} t_{i}(s)\left(\lambda_{i}, u_{i}\right)+t_{n}(s) \frac{\bar{v}-s w(p(\bar{v}))}{\|\bar{v}-s w(p(\bar{v}))\|_{p r}} .
$$

Hence, when $s>0$ is small and $o(s)=o\left(\|p(\bar{v}(s))-p(\bar{v})\|_{p r}\right)$, we have $\bar{v}(s) \in U$ and

$$
\begin{aligned}
& J(p(\bar{v}(s)))-J(p(\bar{v})) \\
= & \langle\nabla J(p(\bar{v})), p(\bar{v}(s))-p(\bar{v})\rangle_{p r}+o(s)=\langle\nabla J(p(\bar{v})), p(\bar{v}(s))\rangle_{p r}+o(s) \\
= & -\operatorname{sign}\left(t_{n}\right) t_{n}(s) s \frac{\langle\nabla J(p(\bar{v})), G(p(\bar{v}))\rangle_{p r}}{\|\bar{v}-s w(p(\bar{v}))\|_{p r}}+o(s) \leq-\frac{1}{4} s \theta\left|t_{n}\right|\|\nabla J(p(\bar{v}))\|_{p r},
\end{aligned}
$$

since $\|p(\bar{v}(s))-p(\bar{v})\|_{p r} \rightarrow 0$ as $s \rightarrow 0,\langle\nabla J(p(\bar{\lambda}, \bar{u})), G(p(\bar{v}))\rangle_{p r} \geq\|\nabla J(p(\bar{v}))\|_{p r}>0$ and $p(\bar{v}) \notin L_{p r}$. $\square$

THEOREM 2.4. Assume that a peak selection $p$ of $J$ is continuous at $\bar{v}=(\bar{\lambda}, \bar{u}) \in$ $U$. If $J(p(\bar{v}))=$ local $-\min _{v \in U} J(p(v))$ and $d\left(p(\bar{v}), L_{p r}\right)>0$, then $p(\bar{v})$ is a critical point of $J$.

If we define a solution set $\mathcal{M}=\{p(v): v \in U\}$, then a local minimum of $J$ on $\mathcal{M}$ leads to a critical point $p\left(v^{*}\right)$, which can be approximated by, e.g., a steepest descent method.

2.2. A Local Minimax Algorithm for Finding Eigensolutions. The following is our local minimax algorithm for finding multiple eigensolutions.

Step 0. Given $\theta \in(0,1), \gamma>0, \varepsilon>0$ and $k=1$. Choose $\left(\lambda_{n}^{1}, v_{n}^{1}\right) \in U$ and $\left(t_{1}^{0}, \ldots, t_{n}^{0}\right) \in \mathbb{R}^{n}$.

Step 1. Compute $\left(\mu_{n}^{1}, u_{n}^{1}\right) \equiv p\left(\lambda_{n}^{1}, v_{n}^{1}\right)=\sum_{i=1}^{n-1} t_{i}^{1}\left(\lambda_{i}, u_{i}\right)+t_{n}^{1}\left(\lambda_{n}^{1}, v_{n}^{1}\right)$ where

$$
\left(t_{1}^{1}, \ldots, t_{n}^{1}\right)=\arg \text { local- } \max _{t_{1}, \ldots, t_{n}} J\left[\sum_{i=1}^{n-1} t_{i}\left(\lambda_{i}, u_{i}\right)+t_{n}\left(\lambda_{n}^{1}, v_{n}^{1}\right)\right]
$$

using initial guess $\left(t_{1}^{0}, \ldots, t_{n}^{0}\right)$.

Step 2. If $\left\|\nabla J\left(\mu_{n}^{k}, u_{n}^{k}\right)\right\| \leq \varepsilon$, then output $\left(\mu_{n}^{k}, u_{n}^{k}\right)$, stop. Otherwise, go to Step 3 .

Step 3. Compute a descent direction $w_{n}^{k}=-\operatorname{sign}\left(t_{n}^{k}\right) \mathcal{P}\left(G_{n}^{k}\right)$, where $G_{n}^{k}$ is a pseudogradient of $J$ at $\left(\mu_{n}^{k}, u_{n}^{k}\right)$ with constant $\theta$.

Step 4. Denote $\left(\lambda_{n}^{k}(s), v_{n}^{k}(s)\right)=\frac{\left(\lambda_{n}^{k}, v_{n}^{k}\right)+s w_{n}^{k}}{\left\|\left(\lambda_{n}^{k}, v_{n}^{k}\right)+s w_{n}^{k}\right\|}$ and

$$
\left(\mu_{n}^{k}(s), u_{n}^{k}(s)\right) \equiv p\left(\lambda_{n}^{k}(s), v_{n}^{k}(s)\right)=\sum_{i=1}^{n-1} t_{i}^{k}(s)\left(\lambda_{i}, u_{i}\right)+t_{n}^{k}(s)\left(\lambda_{n}^{k}(s), v_{n}^{k}(s)\right) \text { where }
$$


$\left(2.30 t_{1}^{k}(s), \ldots, t_{n}^{k}(s)\right)=\arg$ local- $\max _{t_{1}, \ldots, t_{n}} J\left(\sum_{i=1}^{n-1} t_{i}\left(\lambda_{i}, u_{i}\right)+t_{n}\left(\lambda_{n}^{k}(s), v_{n}^{k}(s)\right)\right)$

using the initial guess $\left(t_{1}^{k}, \ldots, t_{n}^{k}\right)$. Let

$$
\begin{aligned}
& s_{n}^{k}=\max _{m \in N}\left\{s=\frac{\gamma}{2^{m}} \mid 2^{m}>\left\|w_{n}^{k}\right\|,\left(\lambda_{n}^{k}(s), v_{n}^{k}(s)\right) \in U\right. \text { s.t. } \\
& \left.J\left(\mu_{n}^{k}(s), u_{n}^{k}(s)\right)-J\left(\mu_{n}^{k}, u_{n}^{k}\right) \leq-\frac{s \theta\left|t_{n}^{k}\right|}{4}\left\|\nabla J\left(\mu_{n}^{k}, u_{n}^{k}\right)\right\|\right\} .
\end{aligned}
$$

Step 5. Set $\left(\lambda_{n}^{k+1}, v_{n}^{k+1}\right)=\left(\lambda_{n}^{k}\left(s_{n}^{k}\right), v_{n}^{k}\left(s_{n}^{k}\right)\right),\left(t_{1}^{k+1}, \ldots t_{n}^{k+1}\right)=\left(t_{1}^{k}\left(s_{n}^{k}\right), \ldots, t_{n}^{k}\left(s_{n}^{k}\right)\right)$, $\left(\mu_{n}^{k+1}, u_{n}^{k+1}\right)=\sum_{i=1}^{n-1} t_{i}^{k+1}\left(\lambda_{i}, u_{i}\right)+t_{n}^{k+1}\left(\lambda_{n}^{k+1}, v_{n}^{k+1}\right), k=k+1$ and go to Step 2.

REMARK 2.2. (a) The algorithm starts with $L_{p r}=\{(0,0)\}$ to find $\left(\lambda_{1}, u_{1}\right)$. Then use $L_{p r}=\left[\left(\lambda_{1}, u_{1}\right)\right]$ to find $\left(\lambda_{2}, u_{2}\right)$. By adding newly found solution $\left(\lambda_{n-1}, u_{n-1}\right)$ to expand $L_{p r}$, one may find $\left(\lambda_{n}, u_{n}\right)$. One may also use a symmetry of the problem to define $L_{p r}$, see [21];

(b) In Step 3, $G_{n}^{k}$ can be computed by using a pseudo-gradient or by following a pseudo-gradient flow. In our numerical implementation when $B=W_{0}^{1, r}(\Omega), r>1$ and $\frac{1}{r}+\frac{1}{q}=1$, we choose $L_{p r}^{\prime}$ as follows: When $r \geq 2, B \subset B^{*}=W_{0}^{1, q}(\Omega)$, it is quite natural to set

$$
L_{p r}^{\prime}=\left\{(\lambda, u) \in \mathbb{R} \times B \mid\left\langle(\lambda, u),\left(\lambda_{i}, u_{i}\right)\right\rangle_{p r}=0, i=1,2, \ldots, n-1\right\} ;
$$

When $1<r<2, u_{1}, u_{2}, \ldots, u_{n-1}$ are usually solutions of some differential equation (see our numerical examples in Sections 3.1 and 3.2). They should be nice functions, or, $u_{i} \in B^{*}=W_{0}^{1, q}(\Omega), i=1,2, \ldots, n-1$. Then, we can use (2.4) again. A practical technique to compute the projection of a pseudo-gradient to $L_{p r}^{\prime}$ will be presented in Section 2.3;

(c) In Step 1, since $L_{p r}$ is finite-dimensional, we may either use a decomposition and normalization to choose an initial $\left(\lambda_{n}^{1}, v_{n}^{1}\right) \in S_{L_{p r}^{\prime}}$ or use observation, among all functions "orthogonal" to $\left\{u_{1}, \ldots, u_{n-1}\right\}$, to choose a normalized $v_{n}^{1}$ with the simplest nodal line structure. It does not have to be exact. Since $\lambda_{n}^{1}$ is a scalar and usually $\lambda_{n}^{1}>$ $\lambda_{n-1}$, it can be selected after several trials. One way to select an initial eigenvalue is to use a necessary condition $\lambda_{n}^{1}=\frac{\left\langle F^{\prime}\left(v_{n}^{1}\right), v_{n}^{1}\right\rangle}{\left\langle G^{\prime}\left(v_{n}^{1}\right), v_{n}^{1}\right\rangle}$. The key is to have $p\left(\lambda_{n}^{1}, v_{n}^{1}\right)$ defined. For example, when $L_{p r}=\{(0,0)\}, v_{1}^{1}$ can be any nonzero function, but a positive one has the simplest nodal line structure. When $L_{p r}=\left[\left(\lambda_{1}, u_{1}\right)\right]$ where $u_{1}$ is positive, among all functions "orthogonal" to $u_{1}$, we choose $v_{2}^{1}$ to be sign-changing and normalized with one nodal line, etc.

(d) Replacing a peak selection $p$ by an $L_{p r^{-}} \perp$ selection $p$ in the algorithm, i.e., (2.2) becomes

$\left(t_{i}^{1}, \ldots, t_{n}^{1}\right)$ solves $\left\langle\left(\lambda_{n}^{1}, v_{n}^{1}\right), \nabla J\left(\mu_{n}^{1}, u_{n}^{1}\right)\right\rangle=0,\left\langle\left(\lambda_{i}, u_{i}\right), \nabla J\left(\mu_{n}^{1}, u_{n}^{1}\right)\right\rangle=0, i=1, \ldots, n-1$,

and (2.3) is replaced by

$$
\begin{aligned}
\left(t_{1}^{k}(s), \ldots, t_{n}^{k}(s)\right) \operatorname{solves} & \left\langle\left(\lambda_{n}^{k}, v_{n}^{k}\right), \nabla J\left(\mu_{n}^{1}(s), u_{n}^{1}(s)\right)\right\rangle=0 \text { and } \\
& \left\langle\left(\lambda_{i}, u_{i}\right), \nabla J\left(\mu_{n}^{1}(s), u_{n}^{1}(s)\right)\right\rangle=0, i=1, \ldots, n-1,
\end{aligned}
$$

a min- $\perp$ algorithm can be designed.

(e) Due to the stepsize rule in Step $4,\left(\lambda_{n}^{1}, u_{n}^{1}\right) \in U$ implies $\left(\lambda_{n}^{k}, u_{n}^{k}\right) \in U$ for all $k=2,3 \ldots$. 
2.3. A Technique to Compute a Pseudo-Gradient. When $B$ is a Hilbert space, we can choose $L_{p r}^{\prime}=L_{p r}^{\perp}$ and $\theta=1$. Then $G_{n}^{k}=\frac{\nabla J\left(\mu_{n}^{k}, u_{n}^{k}\right)}{\left\|\nabla J\left(\mu_{n}^{k}, u_{n}^{k}\right)\right\|}$ is the most efficient pseudo-gradient, i.e., $\theta=1$. To avoid being divided by a term going to the zero, we can use $G_{n}^{k}=\nabla J\left(\mu_{n}^{k}, u_{n}^{k}\right)$ instead. Then we use $w_{n}^{k}=-\operatorname{sign}\left(t_{n}^{k}\right) \nabla J\left(\mu_{n}^{k}, u_{n}^{k}\right)$ and the inequality for the stepsize rule in Step 4 becomes

$$
J\left(\mu_{n}^{k}(s), u_{n}^{k}(s)\right)-J\left(\mu_{n}^{k}, u_{n}^{k}\right) \leq-\frac{s\left|t_{n}^{k}\right|}{4}\left\|\nabla J\left(\mu_{n}^{k}, u_{n}^{k}\right)\right\|^{2} .
$$

Denote $\|\cdot\|_{\ell}$ the norm of $\mathbb{R} \times W_{0}^{1, \ell}$. When $B=W_{0}^{1, r}(\Omega)(r>1$ and $r \neq 2)$, a technique to compute a pseudo-gradient in Step 3 is to use

$$
G_{n}^{k}=\frac{\nabla J\left(\mu_{n}^{k}, u_{n}^{k}\right)}{\left\|\nabla J\left(\mu_{n}^{k}, u_{n}^{k}\right)\right\|_{r}} \text { and check } \gamma_{n}^{k}=\frac{\left\|\nabla J\left(\mu_{n}^{k}, u_{n}^{k}\right)\right\|_{2}^{2}}{\left\|\nabla J\left(\mu_{n}^{k}, u_{n}^{k}\right)\right\|_{r}\left\|\nabla J\left(\mu_{n}^{k}, u_{n}^{k}\right)\right\|_{q}}>\theta_{0}>0 .
$$

If the inequality is satisfied for some $\theta_{0}$ and $\frac{1}{r}+\frac{1}{q}=1$, then $G_{n}^{k}$ is a pseudo-gradient of $J$ at $\left(\mu_{n}^{k}, u_{n}^{k}\right)$ with constant $\theta_{0}$, see [24]. This inequality is satisfied by all of our numerical examples carried out so far. Thus, we can use $w_{n}^{k}=-\operatorname{sign}\left(t_{n}^{k}\right) \nabla J\left(\mu_{n}^{k}, u_{n}^{k}\right)$ and the inequality

$$
J\left(\mu_{n}^{k}(s), u_{n}^{k}(s)\right)-J\left(\mu_{n}^{k}, u_{n}^{k}\right) \leq-\frac{s\left|t_{n}^{k}\right|}{4}\left\|\nabla J\left(\mu_{n}^{k}, u_{n}^{k}\right)\right\|_{2}^{2}
$$

for the stepsize rule in Step 4.

3. Numerical Results of Two Model Problems. In this section, we implement the new algorithm to numerically solve the two model problems. In our computations, linear square or triangular elements are used. Triangular elements are generated basically by a Matlab mesh generator. If a symmetry is used to make our method more efficient (see [21]), a mesh with the same symmetry has to be generated based on the Matlab mesh generator. Finally $\varepsilon=10^{-3}$ is used to terminate an iteration.

3.1. Model Problem 1: A Non Homogeneous NEP. The active Lagrange functional of (1.13) is

$$
J(\lambda, u)=\frac{1}{4} \int_{\Omega}\left[|\nabla u(x)|^{2}+2 V(x) u^{2}(x)+u^{4}(x)\right] d x-\frac{|\lambda|^{k}}{2}\left(\int_{\Omega} u^{2}(x) d x-1\right) .
$$

We first choose $k=2.1, \Omega=(-1,1) \times(-1,1) \subset \mathbb{R}^{2}, \beta=1$ and compute three cases $\gamma_{1}=\gamma_{2}=\frac{\sqrt{2}}{10}, \gamma_{1}=\frac{1}{10}, \gamma_{2}=\frac{\sqrt{2}}{10}$ and $\gamma_{1}=1, \gamma_{2}=\sqrt{2}$ in (1.13).

Since the Frechet derivative $J_{u}^{\prime}(\lambda, u)$ of $J$ in $u$ is in $W^{-1,2}(\Omega)$, to keep the regularity of $W_{0}^{1,2}(\Omega)$, we use its canonical identity $d=\nabla_{u} J(\lambda, u)=\Delta^{-1}\left(J_{u}^{\prime}(\lambda, u)\right) \in$ $W_{0}^{1,2}(\Omega)$. Thus the gradient $\nabla J(\lambda, u)=\left(\nabla_{\lambda} J(\lambda, u), \nabla_{u} J(\lambda, u)\right)$ is used in our algorithm where

$$
\nabla_{\lambda} J(\lambda, u)=-\frac{k}{2} \lambda|\lambda|^{k-2}\left(\int_{\Omega} u^{2} d x-1\right)
$$

and $d=\nabla_{u} J(\lambda, u)$ is solved from

$$
\Delta d=\frac{1}{2} \Delta u-V u-u^{3}+|\lambda|^{k} u, x \in \Omega \text { and }\left.d\right|_{\partial \Omega}=0
$$


since $J_{u}^{\prime}(\lambda, u)=\frac{1}{2} \Delta u-V u-u^{3}+|\lambda|^{k} u$, i.e., for each $\eta \in W_{0}^{1,2}(\Omega)$, we have

$$
\int_{\Omega} J_{u}^{\prime}(\lambda, u)(x) \eta(x) d x=\left.\frac{d}{d t}\right|_{t=0} J(\lambda, u+t \eta)=\int_{\Omega}\left[\frac{1}{2} \Delta u-V u-u^{3}+|\lambda|^{k} u\right] \eta d x .
$$

The profiles of the first seven numerical eigenfunctions and their eigenvalues of each case are displayed in Figs. 3.1-3.3 following an order discussed in Section 4.2. By our numerical computations, this order coincides with the order of eigenvalues, although it has not yet been mathematically proved.

Note that the original Model Problem 1, (1.13) is defined in the domain $\Omega=\mathbb{R}^{2}$ with a "localized" property that $u(x) \rightarrow 0$ as $|x| \rightarrow \infty$. Thus it can be reasonably solved on a sufficiently large bounded domain, e.g., $\Omega_{r}=(-r, r) \times(-r, r)$ with zero Dirichlet boundary condition. Due to the special form of $V(x)=\frac{1}{2}\left(\gamma_{1}^{2} x_{1}^{2}+\gamma_{2}^{2} x_{2}^{2}\right)$, under the transform

$$
w\left(x_{1}, x_{2}\right)=r u\left(r x_{1}, r x_{2}\right),
$$

(1.13) becomes

$$
\lambda w(x)=-\frac{1}{2} \Delta w(x)+U(x) w(x)+\beta w^{3}(x), \quad \forall x \in \Omega_{1}
$$

satisfying the same zero boundary condition on $\Omega_{1}$ and the constraint

$$
\int_{\Omega_{1}} w^{2}(x) d x=1
$$

where $\lambda=r^{2} \mu, U(x)=\frac{1}{2}\left(\alpha_{1}^{2} x_{1}^{2}+\alpha_{2}^{2} x_{2}^{2}\right)$ and $\alpha_{i}=r^{2} \gamma_{i}, i=1,2$. Then the active Lagrange functional under the transform becomes

$$
I(\lambda, w)=\frac{1}{4} \int_{\Omega_{1}}\left[|\nabla w(x)|^{2}+2 U(x) w^{2}(x)+w^{4}(x)\right] d x-\frac{1}{2} \lambda^{k}\left[\int_{\Omega_{1}} w^{2}(x) d x-1\right] .
$$

Then the two active Lagrange functionals satisfy the relation

$$
\left\|\nabla_{w} I(\lambda, w)\right\|_{H_{0}^{1}\left(\Omega_{1}\right)}=r\left\|\nabla_{u} J(\mu, u)\right\|_{H_{0}^{1}\left(\Omega_{r}\right)} .
$$

This relation will be taken into account in the algorithm to control the computational error.

Next to examine the "localized" property of the problem, we solve (1.13) with $\beta=1, \gamma_{1}=\gamma_{2}=0.2$ on $\Omega=\Omega_{r}, r=10$. Under the transform (3.2), we solve (3.3) instead. The profiles of the first six scaled eigenfunctions and their eigenvalues are displayed in Fig. 3.4.

We use $L_{p r}=\{(0,0)\}$ to compute solutions (a)-(f) in Fig. 3.1, solutions (a)-(d), (f)-(g) in Fig. 3.2, solutions (a)-(e), (g) in Fig. 3.3 and solutions (a)-(c) and (e)-(f) in Fig. 3.4. We set $\left.L_{p r}=\left[\left(\lambda_{1}\right)^{\frac{1}{2.1}}, u_{1}\right)\right]$ to capture solution (g) in Fig. 3.1, solutions (e) in Figs. 3.3 and 3.8, solution (f) in Fig. 3.2 and solution (d) in Fig. 3.4, where $\left(\lambda_{1}, u_{1}\right)$ is their first eigensolutions.

The following symmetry of the problem has been used to enhance the efficiency in computing a specific solution: the odd symmetry about the y-axis for solutions in Figs. 3.1 (b), Fig. 3.2 (c) and Fig.3.3 (c); the odd symmetry about the x-axis for solutions in (b) of Figs. 3.2- 3.4; the odd symmetry about the line $y=x$ for solutions in (c) of Figs. 3.1 and 3.4, and (d) of Figs. 3.2 and 3.3; the odd symmetry about 
the x-axis and the y-axis for solutions in Figs. 3.1 (d), Fig. 3.4 (e), Fig. 3.2 (f) and Fig. 3.3 (e); the odd symmetry about lines $y=x$ and $y=-x$ for solutions in (f) of Figs. 3.1 and 3.4, and (g) of Figs. 3.2 and 3.3; the 4-rotation symmetry for solutions in Fig. 3.1 (g), Figs. 3.2 (e), Fig. 3.4 (d) and Fig. 3.3 (f); the local odd symmetry about the lines $x=\frac{1}{3}$ and $x=-\frac{1}{3}$ for solution in Fig. 3.1 (e); Even symmetry about the $x$ and $y$ axes for solution in Fig.3.4 (g).

Following Remark 2.2 (c) about "orthogonality" and nodal lines, selection of an initial guess $v_{n}^{1}$ for computing an eigenfunction by our method can be flexible. To have a desired profile, one can construct $v_{n}^{1}$ by using sine/cosine functions or by solving

$$
-\Delta v_{n}^{1}(x)=c v(x) \text { in } \Omega \text { and } v_{n}^{1}(x)=0 \text { on } \partial \Omega,
$$

where $c v(x)=+/-$ if one wants $v_{n}^{1}$ to be concave up/down at $x \in \Omega$ and $c v(x)=0$ if the concavity of $v_{n}^{1}$ at $x$ is not of concern. For instructional purpose, we list our choices of $c v(x)$ in (3.6) for initial guesses used in computing the first seven eigenfunctions shown in Fig. 3.1. With our numerical results, by comparing (d)-(g) in Figs. 3.1 and

\begin{tabular}{|c|c|c|c|}
\hline$c v(x, y)=$ & Refer & $c v(x, y)=$ & Refer \\
\hline-1 & (a)\&(g) & -1 if $x>0,+1$ if $x<0$ & (b) \\
\hline-1 if $y>x,+1$ if $y<x$ & (c) & -1 if $x y>0,+1$ otherwise & (d) \\
\hline-1 if $|x|>0.15,+1$ otherwise & (e) & -1 if $|x|>|y|,+1$ otherwise & (f) \\
\hline
\end{tabular}

3.4, and (e)-(g) in Figs. 3.2 and 3.3, we noted some pattern order changing phenomena; By comparing Fig. 3.1 with Fig. 3.4, we also noted that when the domain becomes larger, the peaks of the eigenfunctions stay farther away from the boundary and the corner effect of the domain to the eigenfunction profiles becomes less visible. Consequently eigenfunctions (b) and (c), (e) and (f) in Fig.3.4 are virtually the same. This observation illustrates the "localized" property of the problem. Note that the differences in the eigenvalues of Fig.3.4 (d)-(g) are within the computational error and due to a pattern order change among (d)-(g) in Figs.3.1 and 3.4, it is reasonable to believe that the eigenvalues of Fig.3.4 (d)-(g) are actually the same.

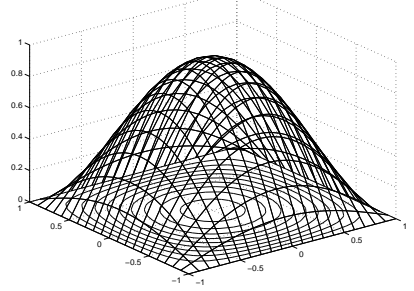

(a)

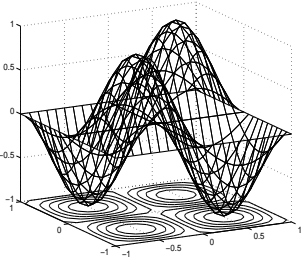

(d)

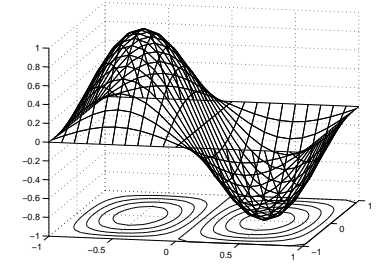

(b)

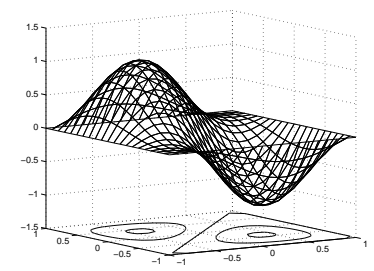

(c)

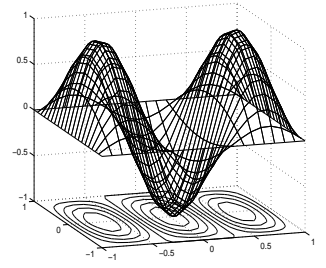

(e)

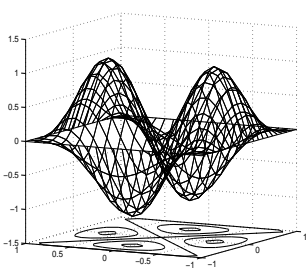

(f)

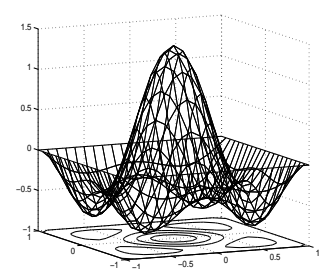

(g)

FIG. 3.1. The first 7 eigenfunctions of (3.3) with $\beta=1, \gamma_{1}=\gamma_{2}=\frac{\sqrt{2}}{10}$ and their eigenvalues (a) 3.0142, (b) 6.7235, (c) 6.8136, (d) 10.4327, (e) 12.8946, (f) 12.9896 and (g) 12.9940. 


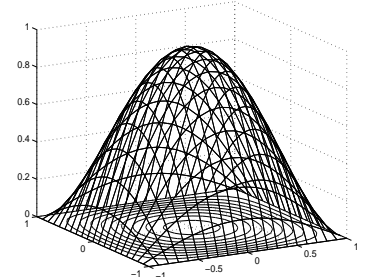

(a)

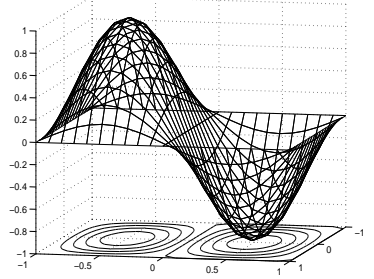

(b)

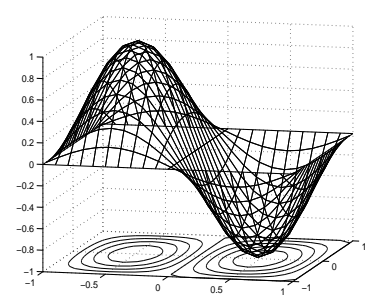

(c)

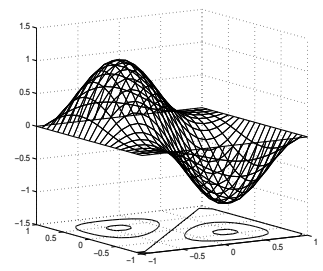

(d)

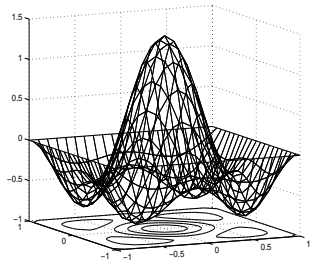

(e)

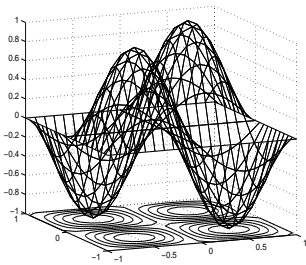

(f)

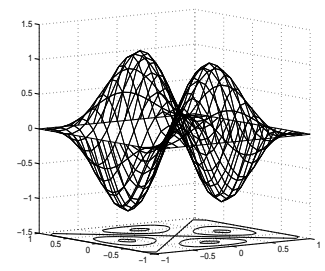

(g)

FIG. 3.2. The first 7 eigenfunctions of (3.3) with $\beta=1, \gamma_{1}=\frac{1}{10}, \gamma_{2}=\frac{\sqrt{2}}{10}$ and their eigenvalues (a) 3.0137, (b) 6.7222 , (c) 6.7230 , (d) 10.4327 , (e) 12.8946 , (f) 12.9896 and (g) 12.9940 .

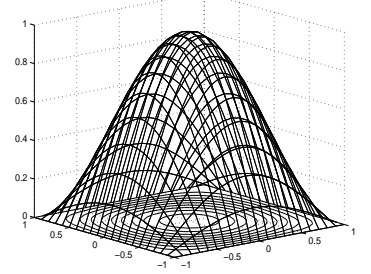

(a)

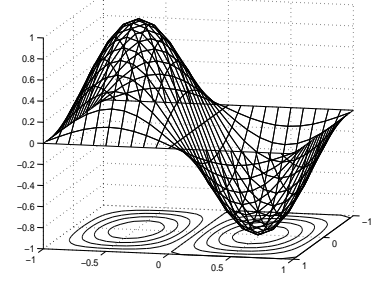

(b)

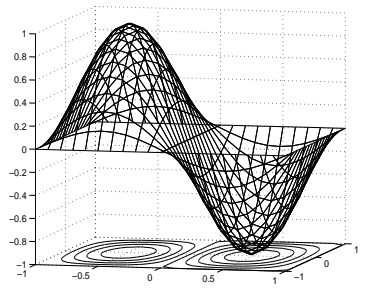

(c)

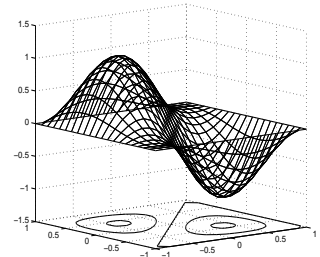

(d)

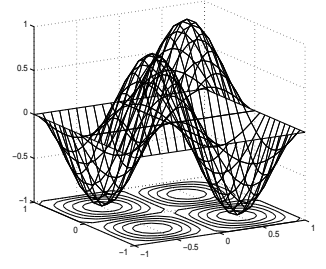

(e)

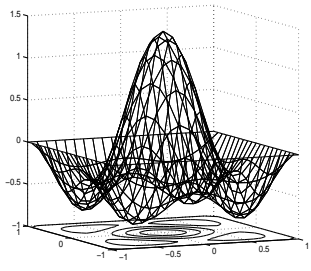

(f)

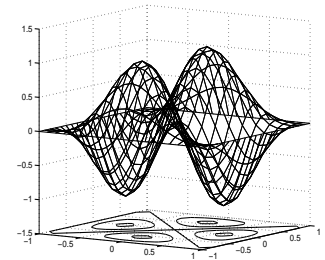

$(\mathrm{g})$

FIG. 3.3. The first 7 eigenfunctions of (3.3) with $\beta=1, \gamma_{1}=1, \gamma_{2}=\sqrt{2}$ and their eigenvalues (a) 3.2210 , (b) 6.9995, (c) 7.0705 , (d) 7.1207 , (e) 10.8492, (f) 13.3125 and (g) 13.3180 . 

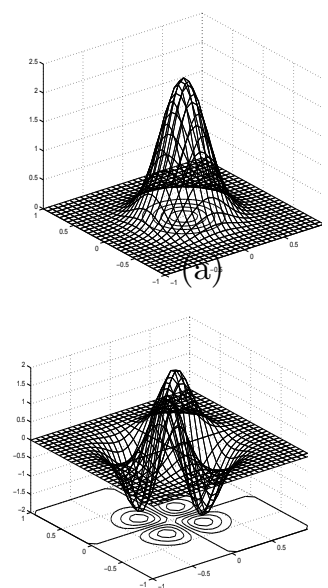

(e)
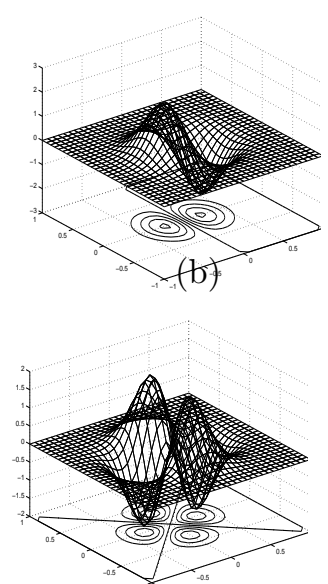

(f)
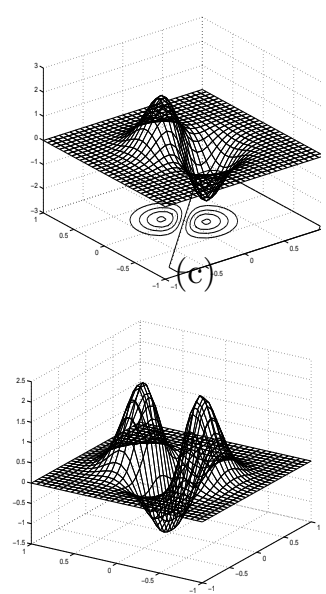

$(\mathrm{g})$
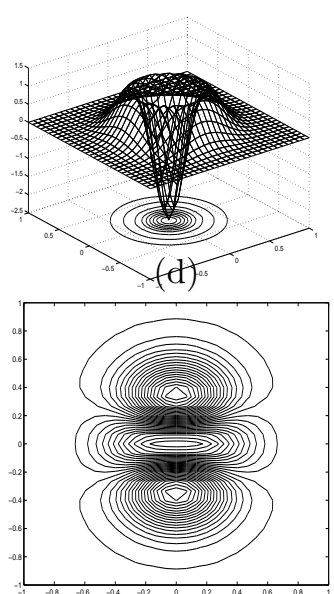

(h)

FIG. 3.4. The first 7 eigenfunctions $w(x)=10 u(10 x)$ of (3.3) with $\beta=1, \gamma_{1}=\gamma_{2}=0.2$ and their eigenvalues $\mu$ (a) 0.229817, (b) 0.422992, (c) 0.422995, (d) 0.615675, (e) 0.617554, (f) 0.617599 and (g) 0.619767. (h) Fine contours of $w$ in $(g)$.

3.2. Model Problem 2: A Homogeneous NEP. The active Lagrange functional of (1.14) for $k \geq 1$ is

$J(\lambda, u)=\frac{1}{p} \int_{\Omega}|\nabla u(x)|^{p} d x-\frac{|\lambda|^{k}}{q}\left(\int_{\Omega} w(x)|u(x)|^{q} d x-1\right),(\lambda, u) \in \mathbb{R} \times\left(W_{0}^{1, p}(\Omega) \backslash\{0\}\right)$. $(3.7)$

The case $p \leq q$ has been solved in $[24,26]$. But the case $q<p$ has not been solved so far, since its standard Lagrange functional (1.15) or (3.7) with $k=1$ fails to have a mountain pass structure. While the new active Lagrange functional (3.7) possesses a mountain pass structure if $p<k+q$. Thus now we are able to solve this case.

To compute a pseudo-gradient, follow the technique stated in Section 2.3, we need to compute the gradient $\nabla J(\lambda, u)=\left(\nabla_{\lambda} J(\lambda, u), \nabla_{u} J(\lambda, u)\right)$ where

$$
\begin{aligned}
\nabla_{\lambda} J(\lambda, u) & =-\frac{k}{q}|\lambda|^{k-2} \lambda\left(\int_{\Omega} w(x)|u(x)|^{q} d x-1\right), \\
\left.d=\nabla_{u} J(\lambda, u)\right) & =\Delta^{-1}\left(J_{u}^{\prime}(\lambda, u)\right) \in W_{0}^{1, q}(\Omega),
\end{aligned}
$$

$J_{u}^{\prime}(\lambda, u) \in W^{-1, q}(\Omega)$ is the Frechet derivative of $J$ in $u$ defined by

$$
\begin{aligned}
\int_{\Omega} J_{u}^{\prime}(\lambda, u)(x) v(x) d x & =\left.\frac{d}{d t}\right|_{t=0} J(\lambda, u+t v) \\
& =\int_{\Omega}\left[\Delta_{p} u(x)+|\lambda|^{k} w(x)|u(x)|^{q-2} u(x)\right] v(x) d x,
\end{aligned}
$$

for each $v \in W_{0}^{1, p}(\Omega)$, i.e., $d=\nabla_{u} J(\lambda, u)$ is solved from

$$
\Delta d(x)=\Delta_{p} u(x)+|\lambda|^{k} w(x)|u(x)|^{q-2} u(x), x \in \Omega \quad \text { and }\left.\quad d\right|_{\partial \Omega}=0 .
$$

The inequality $\gamma_{n}^{k}>\theta_{0}>0$ in Section 2.3 is satisfied in all our numerical computations. Many numerical examples are successfully solved. Here we only present the cases where $p=1.75,2.5, q=2, w(x) \equiv 1$ and $\bar{\Omega} \subset \mathbb{R}^{2}$ is either the square $[-1,1] \times[1,1]$ 
or the unit disk. We use $k=2$ in those cases. For each case, the profiles of the first seven (on the square domain) and the first four (on the unit disk domain) numerical eigenfunctions and their eigenvalues are displayed in Figs. 3.5-3.8 following an order discussed in Section 4.2.

Except the eigensolutions in Figs. 3.5(f), 3.6(e), 3.7(d) and 3.8(d) that are computed by using $L_{p r}=\left[\left(\sqrt{\lambda_{1}}, u_{1}\right)\right]$ and the 4-rotation symmetry, where $\left(\lambda_{1}, u_{1}\right)$ is their first eigensolution, all the other eigensolutions are captured by using $L_{p r}=\{(0,0)\}$ and their symmetries: more specifically, the odd symmetry about the line $y=x$ for Figs. 3.5(b) and 3.6(c); the odd symmetry about the $y$-axis for Figs. 3.5(c), $3.6(\mathrm{~b}), 3.7(\mathrm{~b})$ and $3.8(\mathrm{~b})$; the odd symmetry about the $x$-axis and the $y$-axis for Figs. 3.5(d), 3.6(d), 3.7(c) and 3.8(c); the local odd symmetry about the lines $x= \pm \frac{1}{3}$ for Figs. 3.5(e) and 3.6(g); the odd symmetry about the lines $y= \pm x$ for Figs. $3.5(\mathrm{~g})$ and 3.6(f).

After observing our numerical results, we find some interesting pattern order switching phenomena. For the $p$-Laplacian, when the parameter $p$ changes from $p<2$ across $p=2$ to $p>2$, e.g., $p=1.75$ and $p=2.5$, the pattern of some eigenfunctions switch order, comparing Figs. 3.5(b) (peaks are corner-to-corner) and (c) (peaks are side-to-side) with Figs. 3.6(b) (peaks are side-to-side) and (c) (peaks are corner-tocorner); also comparing patterns in Figs. 3.5 (e), (f), (g) with patterns in Figs. 3.6 (e), (f), (g). These phenomena imply that when $p=2$, the p-Laplacian becomes the usual Laplacian, the second eigensolution is of multiplicity 2 and the 4 th eigensolution is of multiplicity 3.

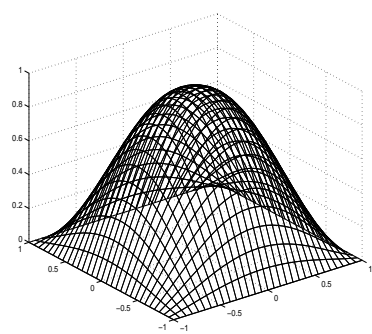

(a)

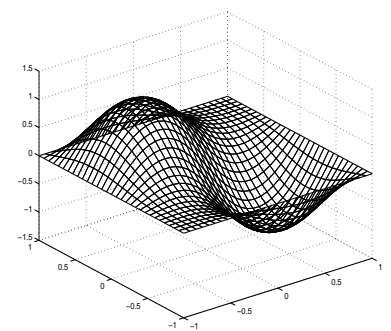

(b)

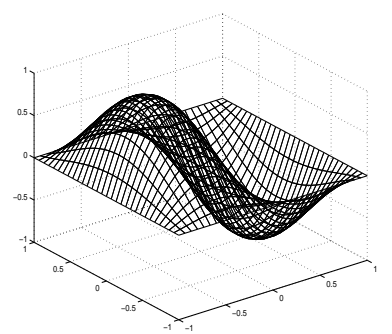

(c)

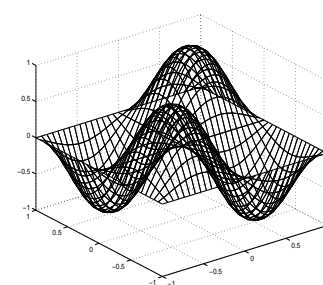

(d)

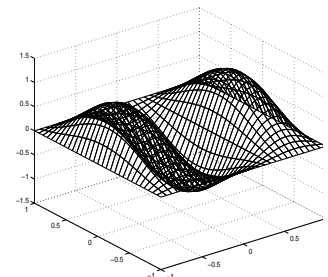

(e)

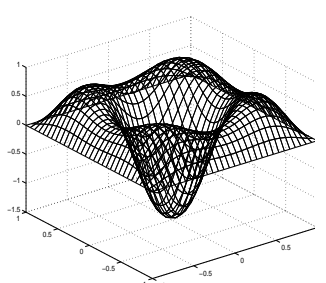

(f)

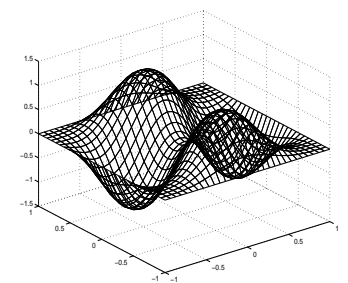

(g)

FIG. 3.5. The first 7 eigenfunctions of (3.7) with $\Omega=(-1,1) \times(-1,1), p=1.75$ and their eigenvalues (a) 4.7189, (b) 10.2698 (peaks are corner to corner), (c) 10.3400 (peaks are side to side), (d) $15.8714,(e) 18.6248,(f) 18.7970$ and $(g) 18.8347$. 


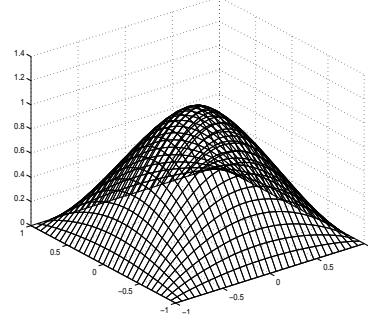

(a)

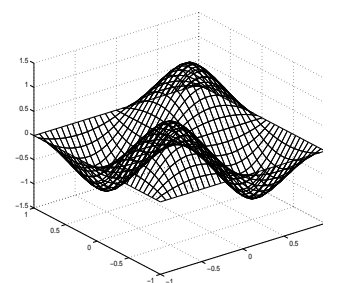

(d)

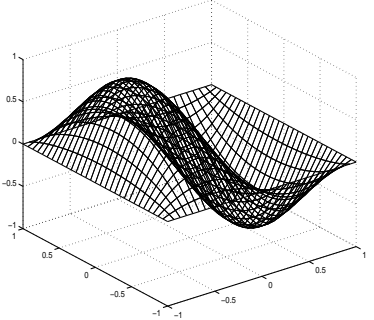

(b)

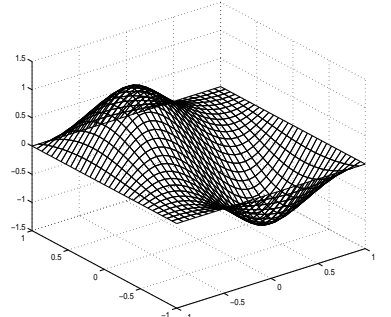

(c)

FIG. 3.6. The first 7 eigenfunctions of (3.7) with $\Omega=(-1,1) \times(-1,1), p=2.5$ and their eigenvalues (a) 5.3876, (b) 17.5278 (peaks are side to side), (c) 17.7931 (peaks are corner to corner), (d) 30.4723, (e) 41.7312, (f) 42.3187 and ( $g$ ) 42.6471.

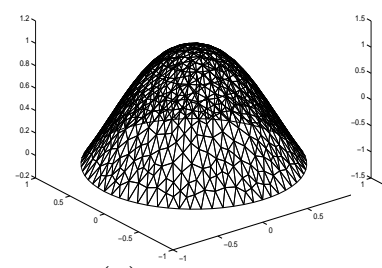

(a)

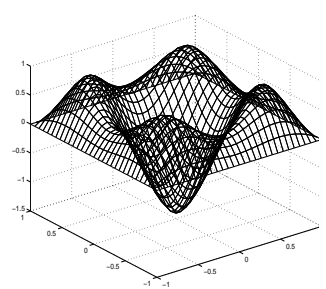

(e)

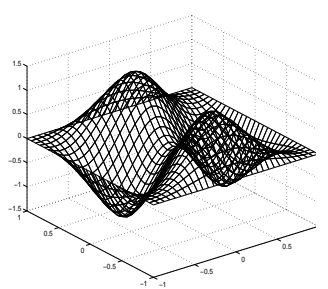

(f)

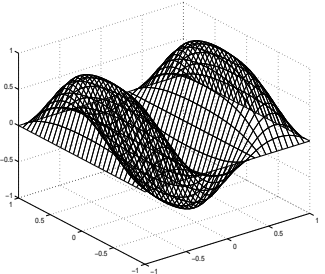

(g)

FIG. 3.7. Eigenfunctions of (3.7) with $\Omega$ the unit disk, $p=1.75$ and their eigenvalues (a) 5.3092, (b) 11.7245, (c) 19.7522 and (d) 21.7686.

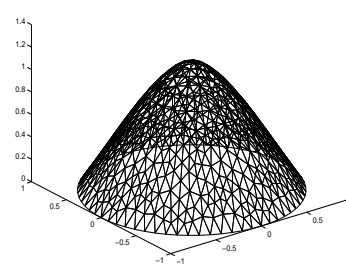

(a)

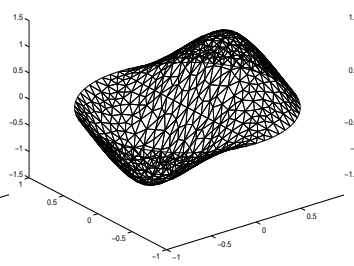

(b)

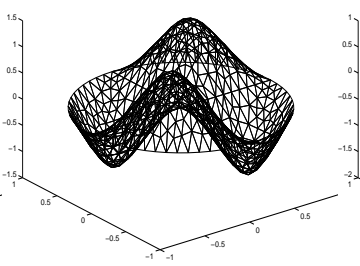

(c)

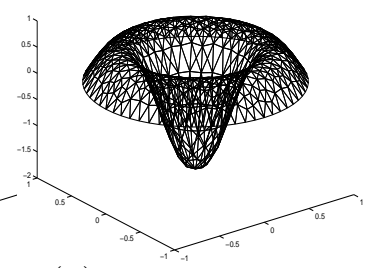

(d)

FIG. 3.8. Eigenfunctions of (3.7) with $\Omega$ the unit disk, $p=2.5$ and their eigenvalues (a) 6.8327 , (b) 22.9888 , (c) 46.9634 and (c) 58.4641.

4. Some Theoretical Results on the Algorithm. Now we present some theoretical results related to our new method. We always assume that $U$ is defined as in Remark 2.1 (c).

4.1. On the Convergence of the Algorithm. The following PS condition is commonly used in critical point theory.

Definition 4.1. A functional $J \in C^{1}(\mathbb{R} \times B, \mathbb{R})$ is said to satisfy the PalaisSmale (PS) condition if for every $\left(\lambda_{n}, w_{n}\right) \in \mathbb{R} \times B$ s.t. $J\left(\lambda_{n}, w_{n}\right)$ is bounded and 
$\nabla J\left(\lambda_{n}, w_{n}\right) \rightarrow 0$, then $\left(\lambda_{n}, w_{n}\right)$ possesses a convergent subsequence.

The following lemmas will be used. Their proofs are similar to those in [25].

Lemma 4.2. $\left\|(\lambda, v)-\frac{(\lambda, v)-(\mu, w)}{\|(\lambda, v)-(\mu, w)\|}\right\| \leq \frac{2\|(\mu, w)\|}{\|(\lambda, v)-(\mu, w)\|}, \quad(\lambda, v) \in \mathbb{R} \times B$, $\|(\lambda, v)\|=1, \forall(\mu, w) \in \mathbb{R} \times B$.

LEMmA 4.3. Let $\left(\lambda_{0}, v_{0}\right) \in U$ and $p$ be an $L_{p r}-\perp$ selection of $J$ defined in $U$. If (1) $p$ is continuous at $\left(\lambda_{0}, v_{0}\right)$ and $G(p(\lambda, v))$ follows a pseudo-gradient flow with constant $\theta \in(0,1)$, or $p$ is locally Lipschitz continuous in a neighborhood of $\left(\lambda_{0}, v_{0}\right)$ and $G(p(\lambda, v))$ is just a pseudo-gradient of $J$ at $p\left(\lambda_{0}, v_{0}\right)$ with constant $\theta \in(0,1)$, (2) $d\left(p\left(\lambda_{0}, v_{0}\right), L_{p r}\right)>0$, and (3) $\nabla J\left(p\left(\lambda_{0}, v_{0}\right)\right) \neq 0$, then there are $\varepsilon>0$ and $s_{0}=\frac{\gamma}{2^{m_{0}}}$, where $m_{0}$ is a positive integer, s.t. $\forall(\lambda, v) \in U$ with $\left\|(\lambda, v)-\left(\lambda_{0}, v_{0}\right)\right\|<\varepsilon$, we have $s_{0}\|\mathcal{P}(G(p(\lambda, v)))\| \leq \gamma$ and

$$
J\left(p\left(\lambda\left(s_{0}\right), v\left(s_{0}\right)\right)\right)-J\left(p(\lambda, v)<-\frac{1}{4} s_{0} \theta\left|t_{(\lambda, v)}\right|\|\nabla J(p(\lambda, v))\|,\right.
$$

where $\left(\lambda\left(s_{0}\right), v\left(s_{0}\right)\right)=\frac{(\lambda, v)-\operatorname{sign}\left(t_{(\lambda, v)}\right) s_{0} \mathcal{P}(G(p(\lambda, v)))}{\left\|(\lambda, v)-\operatorname{sign}\left(t_{(\lambda, v)}\right) s_{0} \mathcal{P}(G(p(\lambda, v)))\right\|}, p(\lambda, v)=t_{(\lambda, v)}(\lambda, v)+$ $w_{(\lambda, v)}$ for some $w_{(\lambda, v)} \in L_{p r}$ and $\gamma \in(0,1)$ is a constant.

Denote $\|\cdot\|_{\ell}$ the norm of $\mathbb{R} \times W_{0}^{1, \ell}(\Omega), \ell>1$.

LEMmA 4.4. Let $B=W_{0}^{1, r}(\Omega)(1<r<2)$ and $\left(\lambda_{0}, v_{0}\right) \in U$. Assume $p$ is a $L_{p r}$ $\perp$ selection of $J$ defined in $U$ s.t. $p$ is continuous at $\left(\lambda_{0}, v_{0}\right)$ and $d\left(p\left(\lambda_{0}, v_{0}\right), L_{p r}\right)>0$. If $\nabla J\left(p\left(\lambda_{0}, v_{0}\right)\right) \neq 0$, then there exists $\varepsilon>0$ and $s_{0}=\frac{\gamma}{2^{m_{0}}}$, where $m_{0}$ is a positive integer, s.t. for every $(\lambda, v) \in U,\left\|(\lambda, v)-\left(\lambda_{0}, v_{0}\right)\right\|<\varepsilon$, we have $s_{0}\|\mathcal{P}(\nabla J(p(\lambda, v)))\| \leq \gamma$ and

$$
J\left(p\left(\lambda\left(s_{0}\right), v\left(s_{0}\right)\right)\right)-J(p(\lambda, v))<-\frac{\left|t_{(\lambda, v)}\right| s_{0}}{4}\|\nabla J(p(\lambda, v))\|_{2}^{2},
$$

where $\left(\lambda\left(s_{0}\right), v\left(s_{0}\right)\right)=\frac{(\lambda, v)-\operatorname{sign}\left(t_{(\lambda, v)}\right) s_{0} \mathcal{P}(\nabla J(p(\lambda, v)))}{\left\|(\lambda, v)-\operatorname{sign}\left(t_{(\lambda, v)}\right) s_{0} \mathcal{P}(\nabla J(p(\lambda, v)))\right\|}, p(\lambda, v)=t_{(\lambda, v)}(\lambda, v)+$ $w_{(\lambda, v)}$ for some $w_{(\lambda, v)} \in L_{p r}$ and $\gamma \in(0,1)$ is a constant.

LEMMA 4.5. Let $B=W_{0}^{1, r}(\Omega)(r>2)$ and $\left(\lambda_{0}, v_{0}\right) \in U$. Assume $p$ is a $L_{p r}-\perp$ selection of $J$ defined in $U$ s.t. $p$ is Lipschitz continuous in a neighborhood of $\left(\lambda_{0}, v_{0}\right)$ and $d\left(p\left(\lambda_{0}, v_{0}\right), L\right)>0$. If $\nabla J\left(p\left(\lambda_{0}, v_{0}\right)\right) \neq 0$, then for any $\left(\lambda_{k}, v_{k}\right) \in$ $U, \lim _{k \rightarrow \infty}\left(\lambda_{k}, v_{k}\right)=\left(\lambda_{0}, v_{0}\right)$ and $\left\|\nabla J\left(p\left(\lambda_{k}, v_{k}\right)\right)\right\|_{r} \leq M$ for some constant $M$, there is $s_{0}=\frac{\gamma}{2^{m_{0}}}$, where $m_{0}$ is a positive integer, s.t. when $k$ is large, we have $s_{0}\left\|\mathcal{P}\left(\nabla J\left(p\left(\lambda_{k}, v_{k}\right)\right)\right)\right\| \leq \gamma$ and

$$
J\left(p\left(\lambda_{k}\left(s_{0}\right), v_{k}\left(s_{0}\right)\right)\right)-J\left(p\left(\lambda_{k}, v_{k}\right)\right)<-\frac{\left|t_{k}\right| s_{0}}{4}\left\|\nabla J\left(p\left(\lambda_{k}, v_{k}\right)\right)\right\|_{2}^{2},
$$

where $\left(\lambda_{k}\left(s_{0}\right), v_{k}\left(s_{0}\right)\right)=\frac{\left(\lambda_{k}, v_{k}\right)-\operatorname{sign}\left(t_{k}\right) s_{0} \mathcal{P}\left(\nabla J\left(p\left(\lambda_{k}, v_{k}\right)\right)\right)}{\left\|\left(\lambda_{k}, v_{k}\right)-\operatorname{sign}\left(t_{k}\right) s_{0} \mathcal{P}\left(\nabla J\left(p\left(\lambda_{k}, v_{k}\right)\right)\right)\right\|}, p\left(\lambda_{k}, v_{k}\right)=t_{k}\left(\lambda_{k}, v_{k}\right)+w_{k}$ for some $w_{k} \in L_{p r}$ and $\gamma \in(0,1)$ is a constant.

We are ready to present a subsequence convergent result.

TheOREM 4.6. Let $J \in C^{1}(\mathbb{R} \times B, \mathbb{R})$ satisfy the $P S$ condition, $p$ be an $L_{p r}$ $\perp$ selection of $J$ defined in $U$ and $\left\{\left(\lambda_{n}^{k}, v_{n}^{k}\right)\right\} \subset U$ be a sequence of points generated by the min- $\perp$ algorithm with $\gamma \in(0,1)$. If (1) $p$ is continuous in $U$ and in Step $2, G_{n}^{k}$ follows a pseudo-gradient flow, or $p$ is locally Lipschitz continuous in $U$ and in Step 2, $G_{n}^{k}$ is just a pseudo-gradient, (2) $\inf _{1 \leq k<\infty} d\left(p\left(\lambda_{n}^{k}, v_{n}^{k}\right), L_{p r}\right) \geq \alpha>0$, (3) 
$\inf _{1 \leq k<\infty} J\left(p\left(\lambda_{n}^{k}, v_{n}^{k}\right)\right)>-\infty$ and (4) $\left\{p\left(\lambda_{n}^{k}, v_{n}^{k}\right)\right\}$ is bounded, then

(a) $\left\{\left(\lambda_{n}^{k}, v_{n}^{k}\right)\right\}_{k=1}^{\infty}$ has a subsequence $\left\{\left(\lambda_{n}^{k_{j}}, v_{n}^{k_{j}}\right)\right\}$ s.t. $p\left(\lambda_{n}^{k_{j}}, v_{n}^{k_{j}}\right) \rightarrow$ a critical point of $J$;

(b) if a subsequence $p\left(\lambda_{n}^{k_{j}}, v_{n}^{k_{j}}\right) \rightarrow\left(\mu_{0}, u_{0}\right)$ as $j \rightarrow \infty$, then $\left(\mu_{0}, u_{0}\right)$ is a critical point of $J$.

Proof. (a) By the stepsize rule, for $k=1,2, \ldots$, we have

$$
J\left(\mu_{n}^{k+1}, u_{n}^{k+1}\right)-J\left(\mu_{n}^{k}, u_{n}^{k}\right) \leq-\frac{1}{4} \theta\left|t_{n}^{k}\right| s_{n}^{k}\left\|\nabla J\left(p\left(\lambda_{n}^{k}, v_{n}^{k}\right)\right)\right\| .
$$

On the other hand, by assumption (2) and Lemma 4.2 , for $k=1,2, \ldots$,

$$
J\left(\mu_{n}^{k+1}, u_{n}^{k+1}\right)-J\left(\mu_{n}^{k}, u_{n}^{k}\right) \leq-\frac{(1-\gamma)}{8} \theta \alpha\left\|\left(\lambda_{n}^{k+1}, v_{n}^{k+1}\right)-\left(\lambda_{n}^{k}, v_{n}^{k}\right)\right\|\left\|\nabla J\left(p\left(\lambda_{n}^{k}, v_{n}^{k}\right)\right)\right\| .
$$

Suppose that there is $\delta>0$ s.t. $\left\|\nabla J\left(p\left(\lambda_{n}^{k}, v_{n}^{k}\right)\right)\right\| \geq \delta$ for any $k$. From (4.2), we have

$$
J\left(\mu_{n}^{k+1}, u_{n}^{k+1}\right)-J\left(\mu_{n}^{k}, u_{n}^{k}\right) \leq-\frac{(1-\gamma)}{8} \theta \alpha \delta\left\|\left(\lambda_{n}^{k+1}, v_{n}^{k+1}\right)-\left(\lambda_{n}^{k}, v_{n}^{k}\right)\right\|, \quad \forall k=1,2, \ldots
$$

Adding up (4.3) and applying assumption (3) give

$$
\begin{aligned}
-\infty & <\lim _{k \rightarrow \infty} J\left(\mu_{n}^{k}, u_{n}^{k}\right)-J\left(\mu_{n}^{1}, u_{n}^{1}\right)=\sum_{k=1}^{\infty}\left[J\left(\mu_{n}^{k+1}, u_{n}^{k+1}\right)-J\left(\mu_{n}^{k}, u_{n}^{k}\right)\right] \\
& \leq-\frac{(1-\gamma)}{8} \theta \alpha \delta \sum_{k=1}^{\infty}\left\|\left(\lambda_{n}^{k+1}, v_{n}^{k+1}\right)-\left(\lambda_{n}^{k}, v_{n}^{k}\right)\right\|
\end{aligned}
$$

i.e., $\left\{\left(\lambda_{n}^{k}, v_{n}^{k}\right)\right\} \subset U$ is a Cauchy sequence. Thus

$$
\left(\lambda_{n}^{k}, v_{n}^{k}\right) \rightarrow(\hat{\lambda}, \hat{v}) \in \bar{U}
$$

Since we assume that $\left\{p\left(\lambda_{n}^{k}, v_{n}^{k}\right)\right\}$ is bounded, by our assumption on $U,(\hat{\lambda}, \hat{v}) \in U$. By the continuity of $p$, we have $\|\nabla J(p(\hat{\lambda}, \hat{v}))\| \geq \delta>0$. Moreover, in view of assumptions (2) and (3), and adding up (4.1), we have

$$
-\infty<\lim _{k \rightarrow \infty} J\left(\mu_{n}^{k}, u_{n}^{k}\right)-J\left(\mu_{n}^{1}, u_{n}^{1}\right) \leq-\frac{1}{4} \theta \alpha \sum_{k=1}^{\infty} s_{k}\left\|\nabla J\left(p\left(\lambda_{n}^{k}, v_{n}^{k}\right)\right)\right\| \leq-\frac{1}{4} \theta \alpha \delta \sum_{k=1}^{\infty} s_{k}
$$

i.e., $s_{k} \rightarrow 0$. It contradicts Lemma 4.3. Therefore, there is a subsequence $\left\{\left(\lambda_{n}^{k_{j}}, v_{n}^{k_{j}}\right)\right\}$ s.t. $\nabla J\left(p\left(\lambda_{n}^{k_{j}}, v_{n}^{k_{j}}\right)\right) \rightarrow 0$ and $\left\{J\left(p\left(\lambda_{n}^{k_{j}}, v_{n}^{k_{j}}\right)\right)\right\}$ is convergent. By the PS condition, $\left\{p\left(\lambda_{n}^{k_{j}}, v_{n}^{k_{i}}\right)\right\}$ has a subsequence that converges to a critical point $\left(\mu_{0}, u_{0}\right)$.

(b) Suppose $\left(\mu_{0}, u_{0}\right)=p\left(\lambda_{0}, v_{0}\right)$ is not a critical point. Then there is $\delta>0$ s.t. $\left\|\nabla J\left(\mu_{n}^{k_{j}}, u_{n}^{k_{j}}\right)\right\|>\delta, j=1,2, \ldots$ By assumption (2) and (4.1), we have

$$
J\left(\mu_{n}^{k_{j}+1}, u_{n}^{k_{j}+1}\right)-J\left(\mu_{n}^{k_{j}}, u_{n}^{k_{j}}\right) \leq-\frac{1}{4} \theta \alpha s_{k_{j}}\left\|\nabla J\left(\mu_{n}^{k_{j}}, u_{n}^{k_{j}}\right)\right\|<-\frac{1}{4} \theta \alpha \delta s_{k_{j}} .
$$

On the other hand, since $\sum_{k=1}^{\infty}\left[J\left(\mu_{n}^{k+1}, u_{n}^{k+1}\right)-J\left(\mu_{n}^{k}, u_{n}^{k}\right)\right]=\lim _{k \rightarrow \infty} J\left(\mu_{n}^{k}, u_{n}^{k}\right)-$ $J\left(\mu_{n}^{1}, u_{n}^{1}\right), \lim _{i \rightarrow \infty}\left(J\left(\mu_{n}^{k_{j}+1}, u_{n}^{k_{j}+1}\right)-J\left(\mu_{n}^{k_{j}}, u_{n}^{k_{j}}\right)\right)=0$. Hence, $\lim _{j \rightarrow \infty} s_{k_{j}}=0$. It contradicts Lemma 4.3. Thus $\left(\mu_{0}, u_{0}\right)$ is a critical point. 
When the technique in Section 2.3 is used, we have the following convergence result.

THEOREM 4.7. Let $B=W_{0}^{1, r}(\Omega)(r>1$ and $r \neq 2)$ where $\Omega$ is bounded when $r>2, J \in C^{1}(\mathbb{R} \times B, \mathbb{R})$ satisfy the $P S$ condition, $p$ be an $L_{p r}-\perp$ selection of $J$ defined in $U$ and $\left\{\left(\lambda_{n}^{k}, v_{n}^{k}\right)\right\} \subset U$ be a sequence generated by the algorithm with $\gamma \in(0,1)$. If (1) $p$ is continuous in $U$ when $1<r<2$ or $p$ is locally Lipschitz continuous in $U$ when $r>2$, (2) $\inf _{1 \leq k<\infty} d\left(p\left(\lambda_{n}^{k}, v_{n}^{k}\right), L_{p r}\right) \geq \alpha>0$, (3) $\inf _{1 \leq k<\infty} J\left(p\left(\lambda_{n}^{k}, v_{n}^{k}\right)\right)>-\infty$, (4) $\left\{p\left(\lambda_{n}^{k}, v_{n}^{k}\right)\right\}$ is bounded and (5) $\gamma_{n}^{k} \geq \theta>0$ when $1<r<2$ or $(5)^{\prime}\left\|\nabla J\left(p\left(\lambda_{n}^{k}, v_{n}^{k}\right)\right)\right\|_{r} \leq$ $M$ for some constant $M>0$ when $r>2$, then

(a) $\left\{\left(\lambda_{n}^{k}, v_{n}^{k}\right)\right\}_{k=1}^{\infty}$ has a subsequence $\left\{\left(\lambda_{n}^{k_{j}}, v_{n}^{k_{j}}\right)\right\}$ s.t. $p\left(\lambda_{n}^{k_{j}}, v_{n}^{k_{j}}\right) \rightarrow$ a critical point of $J$

(b) if a subsequence $p\left(\lambda_{n}^{k_{j}}, v_{n}^{k_{j}}\right) \rightarrow\left(\mu_{0}, u_{0}\right)$ as $j \rightarrow \infty$, then $\left(\mu_{0}, u_{0}\right)$ is a critical point of $J$.

Proof. By the step-size rule, for $k=1,2, \ldots$, we have

$$
J\left(\mu_{n}^{k+1}, u_{n}^{k+1}\right)-J\left(\mu_{n}^{k}, u_{n}^{k}\right) \leq-\frac{1}{4}\left|t_{n}^{k}\right| s_{n}^{k}\left\|\nabla J\left(p\left(\lambda_{n}^{k}, v_{n}^{k}\right)\right)\right\|_{2}^{2}
$$

To $r<2$, by assumption (2) and (5), from (4.5),

$$
J\left(\mu_{n}^{k+1}, u_{n}^{k+1}\right)-J\left(\mu_{n}^{k}, u_{n}^{k}\right) \leq-\frac{1}{4} \theta \alpha s_{n}^{k}\left\|\nabla J\left(p\left(\lambda_{n}^{k}, v_{n}^{k}\right)\right)\right\|_{r}\left\|\nabla J\left(p\left(\lambda_{n}^{k}, v_{n}^{k}\right)\right)\right\|_{q}
$$

By Lemma 4.2, from (4.6),

$J\left(\mu_{n}^{k+1}, u_{n}^{k+1}\right)-J\left(\mu_{n}^{k}, u_{n}^{k}\right) \leq-\frac{(1-\gamma)}{8} \theta \alpha\left\|\left(\lambda_{n}^{k+1}, v_{n}^{k+1}\right)-\left(\lambda_{n}^{k}, v_{n}^{k}\right)\right\|_{r}\left\|\nabla J\left(p\left(\lambda_{n}^{k}, v_{n}^{k}\right)\right)\right\|_{q}$. (4.7)

By using (4.6) and (4.7) instead of (4.1) and (4.2) and Lemma 4.4, conclusion (a) and (b) can be verified in the same way to (a) and (b) of Theorem 4.6 .

To $r>2$, if there is $\delta_{0}>0$ s.t. $\left\|\nabla J\left(p\left(\lambda_{n}^{k}, v_{n}^{k}\right)\right)\right\|_{q} \geq \delta_{0}$ for any $k$, then there is $\delta>0$ s.t. $\left\|\nabla J\left(p\left(\lambda_{n}^{k}, v_{n}^{k}\right)\right)\right\|_{2} \geq \delta$ for any $k$. Thus, by assumption (2) and (5)', from (4.5),

$$
\left.J\left(\mu_{n}^{k+1}, u_{n}^{k+1}\right)-J\left(\mu_{n}^{k}, u_{n}^{k}\right) \leq-\frac{1}{4} \alpha s_{n}^{k} \delta^{2} \leq-\frac{1}{4 M} \alpha s_{n}^{k} \delta^{2} \| \nabla p\left(\lambda_{n}^{k}, v_{n}^{k}\right)\right) \|_{r} .
$$

By Lemma 4.2, from (4.8),

$$
J\left(\mu_{n}^{k+1}, u_{n}^{k+1}\right)-J\left(\mu_{n}^{k}, u_{n}^{k}\right) \leq-\frac{(1-\gamma)}{8 M} \alpha \delta^{2}\left\|\left(\lambda_{n}^{k+1}, v_{n}^{k+1}\right)-\left(\lambda_{n}^{k}, v_{n}^{k}\right)\right\| .
$$

By using (4.8) and (4.9) instead of (4.1) and (4.3) and Lemma 4.5, conclusions (a) and (b) can be verified in the same way as (a) and (b) of Theorem 4.6.

Assumption (4) in Theorems 4.6 and 4.7 is quite natural for any numerical algorithm and can be verified under certain common conditions on $F$ and $G$.

THEOREM 4.8. Let the active Lagrange functional $J(\lambda, u)=F(u)-|\lambda|^{\ell}(G(u)-\alpha)$ where $\alpha>0, \ell>1$ and $F, G \in C^{1}(B, \mathbb{R})$ satisfy the conditions

(a) there is an $m>0$ s.t. $\left\langle G^{\prime}(u), u\right\rangle \geq m G(u)$, and

(b) the term $F(u)-\frac{\left\langle F^{\prime}(u), u\right\rangle}{\ell+m}$ is positive for any $u \neq 0$ and goes to $+\infty$ as $\|u\| \rightarrow+\infty$.

Let $\left(\mu_{n}^{k}, u_{n}^{k}\right)=p\left(\lambda_{n}^{k}, v_{n}^{k}\right),(k=1,2, \ldots)$ be a sequence generated by the algorithm. Then $\left\{\left(\mu_{n}^{k}, u_{n}^{k}\right)\right\}$ is bounded. 
Proof. Since $\left(\mu_{n}^{k}, u_{n}^{k}\right)=p\left(\lambda_{n}^{k}, v_{n}^{k}\right)$ and $p$ is an $L_{p r^{-} \perp}$ selection of $J$,

$$
\begin{aligned}
0 & =\left.\frac{d}{d t} J\left(t\left(\mu_{n}^{k}, u_{n}^{k}\right)\right)\right|_{t=1}=\left\langle F^{\prime}\left(u_{n}^{k}\right), u_{n}^{k}\right\rangle-\ell\left|\mu_{n}^{k}\right|^{\ell}\left(G\left(u_{n}^{k}\right)-\alpha\right)-\left|\mu_{n}^{k}\right|^{\ell}\left\langle G^{\prime}\left(u_{n}^{k}\right), u_{n}^{k}\right\rangle \\
& =\left\langle F^{\prime}\left(u_{n}^{k}\right), u_{n}^{k}\right\rangle-(\ell+m)\left|\mu_{n}^{k}\right|^{\ell}\left(G\left(u_{n}^{k}\right)-\alpha\right)-\left(m \alpha+\left[\left\langle G^{\prime}\left(u_{n}^{k}\right), u_{n}^{k}\right\rangle-m G\left(u_{n}^{k}\right)\right]\right)\left|\mu_{n}^{k}\right|^{\ell}
\end{aligned}
$$

i.e.,

$$
\left|\mu_{n}^{k}\right|^{\ell}\left(G\left(u_{n}^{k}\right)-\alpha\right)=\frac{\left\langle F^{\prime}\left(u_{n}^{k}\right), u_{n}^{k}\right\rangle}{\ell+m}-\frac{m \alpha+\left[\left\langle G^{\prime}\left(u_{n}^{k}\right), u_{n}^{k}\right\rangle-m G\left(u_{n}^{k}\right)\right]}{\ell+m}\left|\mu_{n}^{k}\right|^{\ell} .
$$

Then by our assumptions on $\ell$ and (a), (b), we have

$$
J\left(\mu_{n}^{k}, u_{n}^{k}\right)=F\left(u_{n}^{k}\right)-\frac{\left\langle F^{\prime}\left(u_{n}^{k}\right), u_{n}^{k}\right\rangle}{\ell+m}+\frac{m \alpha+\left[\left\langle G^{\prime}\left(u_{n}^{k}\right), u_{n}^{k}\right\rangle-m G\left(u_{n}^{k}\right)\right]}{\ell+m}\left|\mu_{n}^{k}\right|^{\ell}>0 .
$$

Thus the stepsize rule of the algorithm leads to $0<J\left(\mu_{n}^{k+1}, u_{n}^{k+1}\right)<J\left(\mu_{n}^{k}, u_{n}^{k}\right), k=$ $1,2, \ldots$, or $J\left(\mu_{n}^{k}, u_{n}^{k}\right)$ is bounded. Finally assumption (a) and (b) implies $\left\{\left(\mu_{n}^{k}, u_{n}^{k}\right)\right\}$ is bounded. $\square$ Assumptions (a) and (b) in Theorem 4.8 can be directly verified for the two model problems.

Lemma 4.9. Assumptions (a) and (b) in Theorem 4.8 are satisfied by (3.7) with $\ell=k, m=q$ and $p<k+q$, and by (3.1) with $\ell>m=2$.

Proof. Indeed for (3.7), we have

$$
F(u)-\frac{\left\langle F^{\prime}(u), u\right\rangle}{q+k}=\left(\frac{1}{p}-\frac{1}{q+k}\right) \int_{\Omega}|\nabla u(x)|^{p} d x
$$

and for (3.1), we have $\left\langle F^{\prime}(u), u\right\rangle=2 F(u)+\frac{1}{2} \int_{\Omega} u^{4}(x) d x$ and

$F(u)-\frac{\left\langle F^{\prime}(u), u\right\rangle}{\ell+m}=\frac{\ell+m-2}{4(\ell+m)} \int_{\Omega}\left(|\nabla u(x)|^{2}+2 V(x) u^{2}(x)\right) d x+\frac{\ell+m-4}{4(\ell+m)} \int_{\Omega} u^{4}(x) d x$.

For both (3.7) and (3.1), we have $\left\langle G^{\prime}(u), u\right\rangle=m G(u)$.

A sequence convergence result similar to Theorem 2.1 in [25] can be established.

4.2. On the Order of Eigenfunctions. We first prove that for a homogeneous NEP, its eigenvalues are proportional to their critical values of the active Lagrange functional (1.17).

LEMMA 4.10. For given $\alpha>0$, consider the eigenproblem (1.7) where $F, G$ satisfy the homogeneous condition (1.9) with $m, l>1$. The energy function $J(\lambda, u)=\mathcal{L}(\lambda, u)$ is the active Lagrange functional (1.17). Then, for every eigensolution $\left(\left|\lambda_{0}\right|^{k}, u_{0}\right)$,

$$
J\left(\lambda_{0}, u_{0}\right)=\frac{l \alpha}{m}\left|\lambda_{0}\right|^{k}
$$

Proof. Indeed, we have

$$
F(u)=\int_{0}^{1} \frac{d}{d t} F(t u) d t=\int_{0}^{1}\left\langle F^{\prime}(t u), u\right\rangle d t=\int_{0}^{1} t^{l-1}\left\langle F^{\prime}(u), u\right\rangle d t=\frac{1}{l}\left\langle F^{\prime}(u), u\right\rangle .
$$

Similarly, we have $G(u)=\frac{1}{l}\left\langle G^{\prime}(u), u\right\rangle$. Since $\left(\left|\lambda_{0}\right|^{k}, u_{0}\right)$ is an eigensolution, $F^{\prime}\left(u_{0}\right)-$ $\left|\lambda_{0}\right|^{k} G^{\prime}\left(u_{0}\right)=0$ and $G\left(u_{0}\right)=\alpha$. Thus

$$
J\left(\lambda_{0}, u_{0}\right)=F\left(u_{0}\right)=\frac{1}{m}\left\langle F^{\prime}\left(u_{0}\right), u_{0}\right\rangle=\frac{1}{m}\left\langle\left|\lambda_{0}\right|^{k} G^{\prime}\left(u_{0}\right), u_{0}\right\rangle=\frac{l\left|\lambda_{0}\right|^{k}}{m} G\left(u_{0}\right)=\frac{l \alpha}{m}\left|\lambda_{0}\right|^{k} .
$$


The Morse index is widely used to order nondegenerate saddle points in Hilbert spaces. As its generalization, an order of saddles in Banach spaces is defined in [29] which can handle degenerate saddles and also be used to measure the structural instability of saddle points. Following the same argument as in [29], the following theorem can be proved.

Theorem 4.11. Assume that $B$ is a Banach space, $\left(\lambda_{i}, u_{i}\right), i=1, \ldots, n-1$ are $n-1$ linearly independent eigensolutions, $L_{p r}=\left[\left(\lambda_{1}, u_{1}\right), \ldots,\left(\lambda_{n-1}, u_{n-1}\right)\right]$ and $p$ is a peak selection of $J$ w.r.t. $L_{p r}$ in $U$. If (1) $J(p(\bar{\lambda}, \bar{u}))=\min _{(\lambda, u) \in U} J(p(\lambda, u))$ and $p(\bar{\lambda}, \bar{u})$ is a strict maximum, (2) $d\left(p(\bar{\lambda}, \bar{u}), L_{p r}\right)>0$ and (3) $p$ is differentiable at $(\bar{\lambda}, \bar{u})$, then $p(\bar{\lambda}, \bar{u})$ is a critical point of $J$, whose order of saddles equal to $\operatorname{dim}\left(L_{p r}\right)$.

For a saddle point $\left(\mu_{n}, u_{n}\right)$, its order of saddles $=\operatorname{dim}\left(L_{p r}\right)$ is known before we use LMM and $L_{p r}$ to find the saddle point. Since LMM is a local method, different initial guesses may lead to saddle points with the same order $\operatorname{dim}\left(L_{p r}\right)$ in different branches. In this case, we compare their critical values, i.e., $J$ or $F$ values. We put the one with less $F$ value before the one with larger $F$ value. When the homogeneous condition is satisfied, the critical values are proportional to the eigenvalues by Lemma 4.10. Thus an order of eigensolutions found by LMM can be established and can also be used to measure the structural instability of eigensolutions found by LMM.

As a final remark, we point out that the algorithm developed in this paper is basically a constrained steepest descent method. It can find multiple eigensolutions in a sequential order by properly setting the subspace $L_{\mathrm{pr}}$ and selecting initial guesses. The selection of an initial guess is flexible. It does not have to be sufficiently close to a desired unknown solution. But by the nature of this algorithm, only a linear convergence rate can be expected. Thus this method can be followed, after a few iterations, by a Newton's method [22] or one of the squared-operator iteration methods [23], if applicable, to speed up local convergence.

Acknowledgement: We would like to thank an anonymous referee for providing us with helpful suggestions and many useful references.

\section{REFERENCES}

[1] V. A. Alexshkevich, Y. V. Kaetashov, A. Zeleninia, V. A. Vysloukh, J. P. Torres and L. Torner, Eigenvalue control and switching by fission of multisoliton bound states in planar waveguides, Optics Letters, 29(2004) 483-485.

[2] W. Bao and Q. Du, Computing the ground state solution of Bose-Einstein condensates by normalized gradient flow, SIAM J. Sci. Comp. 25(2004) 1674-1697.

[3] V. Benci, P. D'Avenia and L. Pisani, Solitons in several space dimensions: Derrick's problem and infinitely many solitons, Arch. Rational Mech. Anal., 154(2000) 297-324.

[4] V. Benci, D. Fortunato and L. Pisani, Soliton-like solutions of Lorentz invariant equation in dimension 3, Rev. Math. Phys., 3(1998) 315-344.

[5] M. Cuesta, Eigenvalue Problems for the p-Laplacian with Indefinite weights, Electronic Journal of Differential Equations, 33(2001) 1-9.

[6] J.I.Diaz, Nonlinear Partial Differential Equations and Free Boundaries, vol. I, Elliptic Equations, Research Notes in Mathematics 106, Pitman Publishing, Boston, 1985.

[7] A. Ferrando, M. Zacares, P. F. de Coradoba, D. Binosi and J, A. Monsoriu, Spatial soliton formation in photonic crystal fibers, Optics Express, 11(2003) 452-459.

[8] J.J. Garcia-Ripoll, V. V. Konotop, B. M. Malomed and V.M.Perez-Garcia, A quasi-local GrossPitaevskii equation for attractive Bose-Einstein condensate, Math. Comp.in Simulation, 62(2003) 21-30.

[9] V. M. Perez-Garcia, H. Michinel, J. I. Cirac, M. Lewenstein and P. Zoller, Low Energy Excitations of a Bose-Einstein Condensate: A Time-Dependent Variational Analysis, Phys. Rev. Lett. 77 (1996) 5320.

[10] J.J. Garcia-Ripoll and V. M. Perez-Garcia, Optimizing Schrodinger functionals using Sobolev 
gradients: Applications to quantum mechanics and nonlinear optics, SIAM Sci. Comp., 23(2001) 1316-1334.

[11] E. P. Gross, Structure of a quantized vortex in boson systems, Nuovo Cimento, 20(1961) 454477.

[12] M. Holland and J. Cooper, Expansion of a Bose-Einstein condensate in a harmonic potential, Phys. Rev. A53 (1996) R1954.

[13] S. V. Izbash, O Filtracii V Kropnozernstom Materiale, Izv. Nauchnoissled, Inst. Gidrotechniki (NIIG), Leningrad, USSR, 1931.

[14] Y. Q. Li and Z. L. Liu, Solutions of an elliptic eigenvalue problem involving subcritical or critical exponents, Comm. P.D.E., 26(2001) 2227-2248.

[15] Y. Li and J. Zhou, A minimax method for finding multiple critical points and its applications to semilinear PDEs, SIAM Sci. Comp., 23(2001) 840-865.

[16] Y. Li and J. Zhou, Convergence results of a local minimax method for finding multiple critical points, SIAM Sci. Comp., 24(2002) 840-865.

[17] E. Montefusco and V. Radulescu, Nonlinear eigenvalue problems for quasilinear operators on bounded domains, Nonlinear Diff. Equat. Appl., 8(2001) 481-497.

[18] L.P.Pitaevskii, Vortex lines in an imperfect Bose gas, Soviet Phys. JETP, 13(1961) 451-454.

[19] P. A. Ruprecht, M. J. Holland, and K. Burnett, Time-dependent solution of the nonlinear Schrdinger equation for Bose-condensed trapped neutral atoms, Phys. Rev. A51 (1995) 4704 .

[20] K. Tso, On a Real Monge-Ampére Functional, Invent. Math., 101(1990) 425-448.

[21] Z. Wang and J. Zhou, An efficient and stable method for computing multiple saddle points with symmetries, SIAM J. Num. Anal., 43(2005) 891-907.

[22] Z. Wang and J. Zhou, A Local Minimax-Newton Method for Finding Critical Points with Symmetries, SIAM J. Num. Anal., 42(2004), 1745-1759.

[23] J. Yang and T. I. Lakoba, Universally-convergent squared-operator iteration methods for solitary waves in general nonlinear wave equations, Studies in Appl. Math., 118 (2007) 153-197.

[24] X. Yao and J. Zhou, A Minimax Method for Finding Multiple Critical Points in Banach Spaces and Its Application to Quasilinear Elliptic PDE, SIAM J. Sci. Comput., 26(2005) 17961890.

[25] X. Yao and J. Zhou, Unified Convergence Results on a Minimax Algorithm for Finding Multiple Critical Points in Banach Spaces, SIAM J. Num. Anal., 45 (2007) 1330-1347.

[26] X. Yao and J. Zhou, Numerical Methods for Computing Nonlinear Eigenpairs, Part I. IsoHomogenous cases, SIAM J. Sci. Comp., 29 (2007) 1355-1374.

[27] E. Zeidler, Nonlinear Functional Analysis and Its Applications III, Springer-Verlag, New York, 1985.

[28] J. Zhou, A Local Min-Orthogonal Method for Finding Multiple Saddle Points, J. Math Anal. Appl., 291(2004) 66-81.

[29] J. Zhou, Instability Analysis of Saddle Points by a Local Minimax Method, Math. Comp., 74(2005) 1391-1411. 